\title{
G11.92-0.61 MM1: A Keplerian disc around a massive young proto-O star
}

\author{
J. D. Ilee ${ }^{1 \star}$, C. J. Cyganowski ${ }^{2}$, P. Nazari ${ }^{2}$, T. R. Hunter ${ }^{3}$, C. L. Brogan ${ }^{3}$, \\ D. H. Forgan ${ }^{2}$ and Q. Zhang ${ }^{4}$ \\ ${ }^{1}$ Institute of Astronomy, Madingley Road, Cambridge CB3 OHA, UK \\ ${ }^{2}$ SUPA, School of Physics 85 Astronomy, University of St Andrews, North Haugh, St Andrews, Scotland, KY16 9SS, UK \\ ${ }^{3}$ NRAO, 520 Edgemont Rd, Charlottesville, VA 22903, USA, \\ ${ }^{4}$ Harvard-Smithsonian Center for Astrophysics, Cambridge, MA 02138, USA
}

Accepted 2016 July 29. Received 2016 July 29; in original form 2016 April 22

\begin{abstract}
The formation process of massive stars is not well understood, and advancement in our understanding benefits from high resolution observations and modelling of the gas and dust surrounding individual high-mass (proto)stars. Here we report sub-arcsecond ( $\$ 1550 \mathrm{au}$ ) resolution observations of the young massive star G11.92-0.61 MM1 with the SMA and VLA. Our $1.3 \mathrm{~mm}$ SMA observations reveal consistent velocity gradients in compact molecular line emission from species such as $\mathrm{CH}_{3} \mathrm{CN}, \mathrm{CH}_{3} \mathrm{OH}$, OCS, $\mathrm{HNCO}, \mathrm{H}_{2} \mathrm{CO}$, DCN and $\mathrm{CH}_{3} \mathrm{CH}_{2} \mathrm{CN}$, oriented perpendicular to the previouslyreported bipolar molecular outflow from MM1. Modelling of the compact gas kinematics suggests a structure undergoing rotation around the peak of the dust continuum emission. The rotational profile can be well fit by a model of a Keplerian disc, including infall, surrounding an enclosed mass of $\sim 30-60 \mathrm{M}_{\odot}$, of which $2-3 \mathrm{M}_{\odot}$ is attributed to the disc. From modelling the $\mathrm{CH}_{3} \mathrm{CN}$ emission, we determine that two temperature components, of $\sim 150 \mathrm{~K}$ and $230 \mathrm{~K}$, are required to adequately reproduce the spectra. Our 0.9 and $3.0 \mathrm{~cm}$ VLA continuum data exhibit an excess above the level expected from dust emission; the full centimetre-submillimetre wavelength spectral energy distribution of MM1 is well reproduced by a model including dust emission, an unresolved hypercompact $\mathrm{H}$ II region, and a compact ionised jet. In combination, our results suggest that MM1 is an example of a massive proto-O star forming via disc accretion, in a similar way to that of lower mass stars.
\end{abstract}

Key words: stars: massive - stars: pre-main-sequence - stars: protostars - submillimetre: stars - radio continuum: stars - stars: individual: G11.92-0.61

\section{INTRODUCTION}

Massive stars $\left(M_{\star}>8 M_{\odot}\right)$ have a very short pre-mainsequence phase $\left(\sim 10^{4}-\right.$ a few $\times 10^{5}$ years, e.g. Davies et al. 2011; Mottram et al. 2011), meaning that they spend the entirety of their formation stages deeply embedded in their parent molecular clouds. Such short formation timescales also mean that there are far fewer examples of young, massive stars when compared with their lower mass counterparts. This inherent scarcity means that young massive stars are, on average, located in much more distant star forming regions $(>1 \mathrm{kpc})$. All of these factors contribute to the ex-

^ Contact e-mail: jdilee@ast.cam.ac.uk treme difficulty of observing young massive stars directly. As a result, their formation mechanisms are poorly understood.

In particular, the process by which young massive stars accrete their high masses is not known. There is little time to accumulate such large masses during their short formation timescales, and accretion onto the central object may be halted or substantially reduced by energetic feedback processes (such as high radiation pressures, strong stellar winds and ionising radiation). Models have suggested that channelling material through a circumstellar accretion disc can overcome these feedback mechanisms (e.g. Yorke \& Sonnhalter 2002; Krumholz et al. 2009; Kuiper et al. 2010, 2011; Harries et al. 2014; Klassen et al. 2016).

Observationally, however, it is not yet clear whether circumstellar accretion discs surround massive young stel- 
lar objects (MYSOs) of all masses and evolutionary stages. In particular, convincing candidates for Keplerian discs around embedded O-type (proto)stars are proving to be particularly elusive (e.g. Cesaroni et al. 2007; Wang et al. 2012; Beltrán \& de Wit 2016, and references therein). Infrared (IR) interferometry (Kraus et al. 2010; Boley et al. 2013) and high-resolution near-infrared spectroscopy (Bik \& Thi 2004; Davies et al. 2010; Wheelwright et al. 2010; Ilee et al. 2013, 2014) have revealed discs on scales of less than $1000 \mathrm{au}$ around MYSOs, but these techniques are limited to relatively evolved, IR-bright objects. Longerwavelength interferometric observations allow access to the circum(proto)stellar environments of less evolved, more embedded MYSOs, but often probe larger spatial scales. In many cases, velocity gradients detected in millimetre and centimetre-wavelength molecular line observations trace 'toroids' - large-scale ( 1000 s to $\gtrsim 10,000 \mathrm{au}$ ), massive $\left(\mathrm{M}_{\text {toroid }} \geq \mathrm{M}_{\star}\right)$, non-equilibrium rotating structures (e.g. Cesaroni 2005; Cesaroni et al. 2006, 2007; Beuther \& Walsh 2008; Beltrán et al. 2011; Cesaroni et al. 2011; Johnston et al. 2014, and references therein). The clustered nature of massive star formation also complicates the search for accretion disc candidates, as multiplicity and rotation can both produce velocity gradients (c.f. Patel et al. 2005; Brogan et al. 2007; Comito et al. 2007), and very high angular resolution observations are required to distinguish between these scenarios.

Searching for small-scale Keplerian accretion discs around embedded distant MYSOs requires sub-arcsecondresolution observations with (sub)millimetre interferometers. Recent studies of this type have yielded a handful of candidate Keplerian discs around O-type (proto)stars (Jiménez-Serra et al. 2012; Qiu et al. 2012; Wang et al. 2012; Hunter et al. 2014; Johnston et al. 2015; Zapata et al. 2015), as well as adding to the sample of good candidates for Keplerian discs around B-type (proto)stars (e.g. Sánchez-Monge et al. 2013; Beltrán et al. 2014; Cesaroni et al. 2014).

In this paper, we report sub-arcsecond resolution Submillimeter Array (SMA) observations of a candidate disc around a high-mass (proto)star, G11.92-0.61 MM1 (hereafter MM1), identified in the course of our studies of GLIMPSE Extended Green Objects (EGOS; Cyganowski et al. 2008). MM1 is one of three compact millimetre continuum cores detected in thermal dust emission in our initial SMA $1.3 \mathrm{~mm}$ observations of the EGO G11.92-0.61 (Cyganowski et al. 2011a, resolution $\sim 2.4^{\prime \prime}$ ), which is located in an infrared dark cloud (IRDC) $\sim 1^{\prime} \mathrm{SE}$ of the more evolved massive star-forming region IRAS 18110-1854. The three members of the G11.92-0.61 (proto)cluster are only resolved at (sub)millimetre and longer wavelengths (Cyganowski et al. 2011a, 2014); the total luminosity of the region is $\sim 10^{4} L_{\odot}$ (Cyganowski et al. 2011a; Moscadelli et al. 2016). MM1 exhibits copious molecular line emission from hot-core molecules (Cyganowski et al. 2011a, 2014) and is coincident with a $6.7 \mathrm{GHz}$ Class II $\mathrm{CH}_{3} \mathrm{OH}$ maser (Cyganowski et al. 2009) and strong $\mathrm{H}_{2} \mathrm{O}$ masers (Hofner \& Churchwell 1996; Breen \& Ellingsen 2011; Sato et al. 2014; Moscadelli et al. 2016), all indicative of the presence of a massive (proto)star. MM1 also has a weak $(\lesssim 1 \mathrm{mJy}) \mathrm{cm}-$ wavelength counterpart, CM1, first detected at $1.3 \mathrm{~cm}$ by Cyganowski et al. (2011b, 2014) and recently also at 4.8 and
$2.3 \mathrm{~cm}$ by Moscadelli et al. (2016) as part of a Very Large Array (VLA) survey of BeSSeL $\mathrm{H}_{2} \mathrm{O}$ maser sources.

Cyganowski et al. (2011a) detected a single dominant bipolar molecular outflow towards the G11.92-0.61 millimetre (proto)cluster, driven by MM1 and traced by wellcollimated, high-velocity ${ }^{12} \mathrm{CO}(2-1)$ and $\mathrm{HCO}^{+}(1-0)$ emission. Near-infrared $\mathrm{H}_{2}$ emission (Lee et al. 2012, 2013) and $44 \mathrm{GHz}$ Class I $\mathrm{CH}_{3} \mathrm{OH}$ masers (Cyganowski et al. 2009) also trace shocked outflow gas. On small $(<1000 \mathrm{au})$ scales, the $\mathrm{H}_{2} \mathrm{O}$ maser proper motions indicate a collimated NE-SW flow, consistent with the orientation of the large-scale molecular outflow (Cyganowski et al. 2011a, 2014; Moscadelli et al. 2016 ). In our $\sim 2.4^{\prime \prime}$-resolution SMA data, the compact, hotcore molecular line emission associated with MM1 displays a velocity gradient oriented roughly perpendicular to the outflow axis, leading Cyganowski et al. (2011a) to suggest an unresolved disc as a possible explanation, but requiring higher angular resolution data for confirmation.

In this paper, we present sub-arcsecond-resolution line and continuum observations of G11.92-0.61 obtained with the highest angular resolution possible with the SMA at $1.3 \mathrm{~mm}$. Together with new VLA subarcsecond-resolution centimetre continuum observations, we use the SMA data to study the molecular gas kinematics of MM1 and to constrain the nature of the central source. Our observations are summarised in Section 2, and our results in Section 3. Section 4 presents our modelling of the kinematics and physical properties of the candidate disc and of the centimetre-(sub)millimetre wavelength emission from the central source, and discusses our results. Our conclusions are summarised in Section 5. We adopt the maser parallax distance to G11.92-0.61 of $3.37_{-0.32}^{+0.39} \mathrm{kpc}$ throughout (Sato et al. 2014)

\section{OBSERVATIONS}

\subsection{Submillimeter Array (SMA)}

The SMA $1.3 \mathrm{~mm}$ Very Extended configuration (VEX) dataset is described in Cyganowski et al. (2014), which uses these data to study G11.92-0.61 MM2, a candidate massive prestellar core located $\sim 7$ '. 2 from MM1 in the G11.920.61 millimetre (proto)cluster. The SMA $1.3 \mathrm{~mm}$ observations were taken in good weather, with $\tau_{225 \mathrm{GHz}} \sim 0.05$ and system temperatures at source transit $\mathrm{T}_{\text {sys }} \sim 90 \mathrm{~K}$; eight antennas were available for the observations. Key observational parameters are listed in Table 1 . The $1.3 \mathrm{~mm}$ line data were resampled to a common velocity resolution of $1.12 \mathrm{~km} \mathrm{~s}^{-1}$, then Hanning-smoothed. The projected baselines ranged from $\sim 20-394 \mathrm{k} \lambda$, corresponding to a largest angular scale (for sensitivity to smooth emission) of $\sim 9^{\prime \prime}$ (see also Cyganowski et al. 2014). We carefully identified the line-free portions of the spectrum in order to perform continuum subtraction and to generate a continuum image with minimal line contamination. The effective bandwidth of the continuum image is approximately two thirds of the total observed bandwidth. All measurements were made from images corrected for the primary beam response.

To measure the position angle of the bipolar molecular outflow driven by MM1, we make use of the ${ }^{12} \mathrm{CO}\left(3_{-}\right.$ 2) line included in the $0.88 \mathrm{~mm}$ SMA dataset presented in 
Table 1. Parameters of the SMA and VLA observations.

\begin{tabular}{|c|c|c|c|c|}
\hline Parameter & SMA $1.3 \mathrm{~mm}$ & SMA $0.88 \mathrm{~mm}$ & VLA $3 \mathrm{~cm}$ & VLA $0.9 \mathrm{~cm}$ \\
\hline Observing date (UT) & 2011 Aug 28 & 2011 Aug 19 & 2015 Jun 25 & 2015 Feb $9-10$ \\
\hline Project code & 2011A-S076 & 2011A-S076 & $15 \mathrm{~A}-232$ & $15 \mathrm{~A}-232$ \\
\hline Configuration & Very Extended & Extended & $\mathrm{A}$ & $\mathrm{B}$ \\
\hline \multicolumn{5}{|l|}{ Phase Center (J2000): } \\
\hline R.A. & $18^{\mathrm{h}} 13^{\mathrm{m}} 58^{\mathrm{s}} .10$ & $18^{\mathrm{h}} 13^{\mathrm{m}} 58^{\mathrm{s}} .10$ & $18^{\mathrm{h}} 13^{\mathrm{m}} 58^{\mathrm{s}} \cdot 10$ & $18^{\mathrm{h}} 13^{\mathrm{m}} 58^{\mathrm{s}} .10$ \\
\hline Dec. & $-18^{\circ} 54^{\prime} 16^{\prime \prime} 7$ & $-18^{\circ} 54^{\prime} 16^{\prime \prime} 7$ & $-18^{\circ} 54^{\prime} 16^{\prime \prime} 7$ & $-18^{\circ} 54^{\prime} 16^{\prime \prime} 7$ \\
\hline Primary beam size (FWHP) & $52^{\prime \prime}$ & $34^{\prime \prime}$ & $4^{\prime}$ & $1.3^{\prime}$ \\
\hline \multicolumn{5}{|l|}{ Frequency coverage: } \\
\hline Lower band (LSB) center & $218.9 \mathrm{GHz}$ & $335.6 \mathrm{GHz}$ & $9 \mathrm{GHz}$ & $31 \mathrm{GHz}$ \\
\hline Upper band (USB) center & $230.9 \mathrm{GHz}$ & $347.6 \mathrm{GHz}$ & $11 \mathrm{GHz}$ & $35 \mathrm{GHz}$ \\
\hline Bandwidth & $2 \times 4 \mathrm{GHz}$ & $2 \times 4 \mathrm{GHz}$ & $2 \times 2.048 \mathrm{GHz}$ & $4 \times 2.048 \mathrm{GHz}$ \\
\hline Subbands & $\mathrm{n} / \mathrm{a}$ & $\mathrm{n} / \mathrm{a}$ & $2 \times 16$ & $4 \times 16$ \\
\hline Channel spacing & $0.8125 \mathrm{MHz}$ & $0.8125 \mathrm{MHz}$ & $\begin{array}{c}1 \mathrm{kHz} \\
\left(30 \mathrm{~km} \mathrm{~s}^{-1}\right)\end{array}$ & $\begin{array}{c}1 \mathrm{kHz} \\
\left(9 \mathrm{~km} \mathrm{~s}^{-1}\right)\end{array}$ \\
\hline Gain calibrator(s) & J1733-130, J1924-292 & J1733-130, J1924-292 & J1832-2039 & J1832-2039 \\
\hline Bandpass calibrator & $3 \mathrm{C} 84$ & $3 \mathrm{C} 84$ & J1924-2914 & J1924-2914 \\
\hline Flux calibrator & Callisto $^{a}$ & Callisto $^{a}$ & $\mathrm{~J} 1331+3030$ & $\mathrm{~J} 1331+3030$ \\
\hline Synthesised beam ${ }^{b}$ & $\begin{array}{l}0^{\prime} .57 \times 0^{\prime} .37 \\
\left(\text { P.A. }=30^{\circ}\right)\end{array}$ & $\begin{array}{l}0^{\prime \prime} 80 \times 0^{\prime \prime} 70 \\
\left(\text { P.A. }=54^{\circ}\right)\end{array}$ & $\begin{array}{l}0^{\prime} 30 \times 0^{\prime} 117 \\
\left(\text { P.A. }=0^{\circ}\right)\end{array}$ & $\begin{array}{c}0 . ' 31 \times 0^{\prime} \cdot 17 \\
\left(\text { P.A. }=-5^{\circ}\right)\end{array}$ \\
\hline Continuum rms noise $^{b}$ & 0.7 mJy beam ${ }^{-1}$ & $3 \mathrm{mJy}_{\text {beam }^{-1}}$ & $6.1 \mu \mathrm{Jy}_{\mathrm{beam}}{ }^{-1}$ & $7.6 \mu \mathrm{Jy}^{\text {beam }^{-1}}$ \\
\hline Spectral line rms noise ${ }^{c}$ & $23 \mathrm{mJy}$ beam $^{-1}$ & 55 mJy beam ${ }^{-1}\left({ }^{12} \mathrm{CO}\right)$ & $\mathrm{n} / \mathrm{a}$ & $\mathrm{n} / \mathrm{a}$ \\
\hline
\end{tabular}

a: Using Butler-JPL-Horizons 2012 models.

$b$ : SMA: for combined LSB+USB continuum image (Briggs weighting, robust $=0.5$ ).

$c$ : Typical rms per channel; Hanning-smoothed. For ${ }^{12} \mathrm{CO}(3-2), \mathrm{rms}$ is per smoothed $3 \mathrm{~km} \mathrm{~s}^{-1}$ channel (see also

Cyganowski et al. 2014).

Cyganowski et al. (2014). Details of the SMA $0.88 \mathrm{~mm}$ observations are included in Table 1 for completeness; here, we consider only the ${ }^{12} \mathrm{CO}(3-2)$ data.

\subsection{Jansky Very Large Array (VLA)}

In 2015, we observed G11.92-0.61 with the NRAO Karl G. Jansky Very Large Array (VLA) in two bands (X and Ka, $3 \mathrm{~cm}$ and $0.9 \mathrm{~cm}$ ) under project code $15 \mathrm{~A}-232$. The observations were taken with dual circular polarization, with onsource observing times of 39 minutes and 86 minutes at 3 and $0.9 \mathrm{~cm}$, respectively. Further details of the observations are given in Table 1 . Due to the broad bandwidth of the datasets, we imaged them using 2 Taylor terms to account for the spectral index of the emission and performed phase-only self-calibration. Due to the proximity of the cometary ultra-compact (UC) H II region G11.94-0.62 (Wood \& Churchwell 1989) about 1' to the north-northeast, we needed to include that source in the model. The X-band image was made with a minimum uv distance of $1300 \mathrm{k} \lambda$ in order to remove ripple from G11.94-0.62 in the vicinity of G11.92-0.61. Our previously-published VLA observations in K-band (1.2 cm; Cyganowski et al. 2014) are also used in modelling the spectral energy distribution in Section 4.3.

\section{RESULTS}

\subsection{Continuum emission}

Figure 1 shows our new VLA centimetre-wavelength continuum images overlaid on the $1.3 \mathrm{~mm}$ SMA VEX image from Cyganowski et al. (2014); observed centimetre continuum properties for CM1 are listed in Table 2. The continuum emission from CM1 is compact at all wavelengths. The fitted centroid positions for CM1 in the 3.0 and $0.9 \mathrm{~cm}$ images are consistent within $<0$ '. 01 , and are within $\sim 0{ }^{\prime} .05$ of the $1.3 \mathrm{~mm}$ MM1 peak (fitted centroid position, Table 2 of Cyganowski et al. 2014). CM1 is unresolved in our deep VLA images, indicating that the source of centimetre-wavelength emission is smaller than our $\sim 1000 \times 570$ au beam. This result is consistent with recent VLA observations at $4.8,2.3$ and $1.4 \mathrm{~cm}$ (resolution 0!32, 0!'19, and 0!'1, respectively): in all cases, the fitted source size is less than the size of the synthesised beam (Moscadelli et al. 2016, their Table 3).

\subsection{Compact molecular line emission: MM1}

In our SMA $1.3 \mathrm{~mm}$ VEX observations, the molecular line emission detected towards G11.92-0.61 consists primarily of compact emission, coincident with the MM1 (sub)millimetre continuum source, from species characteristic of hot cores (including $\mathrm{CH}_{3} \mathrm{CN}$, OCS, $\mathrm{CH}_{3} \mathrm{CH}_{2} \mathrm{CN}$, and $\mathrm{HC}_{3} \mathrm{~N}$; see also Figure 2 of Cyganowski et al. 2014). From the $8 \mathrm{GHz}$ of bandwidth observed with the SMA at $1.3 \mathrm{~mm}$, we identified 31 lines from 10 different chemical species that are potentially strong and unblended enough to be used to study the kinematics of MM1. Details for these lines are given in Table 3 , ordered by decreasing $\mathrm{E}_{\text {upper }}$. For all lines included in Table 3, we created and inspected integrated intensity (moment 0 ) and velocity (moment 1 ) maps. The moment 1 maps were constructed using an intensity threshold of $5 \sigma$, and the velocity extent was chosen to avoid contamination from nearby lines where possible.

Figures 2 and 3 present moment 1 maps for selected 
Table 2. Observed continuum emission properties.

\begin{tabular}{|c|c|c|c|c|c|c|c|}
\hline \multirow[t]{2}{*}{ Obs. $\lambda$} & \multicolumn{2}{|c|}{$\begin{array}{c}\mathrm{J} 2000 \\
\text { Coordinates }^{a}\end{array}$} & \multirow{2}{*}{$\begin{array}{c}\text { Peak } \\
\text { Intensity }^{a} \\
\left(\mathrm{mJy} \mathrm{beam}^{-1}\right)\end{array}$} & \multirow{2}{*}{$\begin{array}{c}\text { Integrated } \\
\text { Flux Density }^{a} \\
(\mathrm{mJy})\end{array}$} & \multirow{2}{*}{$\begin{array}{l}\text { Size }^{a} \\
\left({ }^{\prime \prime} \times{ }^{\prime \prime}\right)\end{array}$} & \multirow{2}{*}{$\begin{array}{c}\text { Size }^{a} \\
(\mathrm{au} \times \mathrm{au})\end{array}$} & \multirow{2}{*}{$\begin{array}{l}\text { P.A. } \\
\left({ }^{\circ}\right)\end{array}$} \\
\hline & $\alpha(\mathrm{h} \mathrm{m} \mathrm{s})$ & $\delta\left({ }^{\circ}{ }^{\prime \prime \prime \prime}\right)$ & & & & & \\
\hline $1.3 \mathrm{~mm}$ & 181358.1099 & -185420.141 & $90.0 \pm 1.0$ & $138.0 \pm 2.0$ & $0.34 \pm 0.01 \times 0.29 \pm 0.01$ & $1150 \times 960$ & $125 \pm 7$ \\
\hline $0.9 \mathrm{~cm}$ & 181358.1108 & -185420.185 & $0.548 \pm 0.011$ & $0.715 \pm 0.023$ & $0.16 \pm 0.02 \times 0.10 \pm 0.01$ & $550 \times 330$ & $160 \pm 12$ \\
\hline $3.0 \mathrm{~cm}$ & 181358.1113 & -185420.191 & $0.203 \pm 0.009$ & $0.161 \pm 0.014$ & $\ldots$ & $\ldots$ & $\ldots$ \\
\hline
\end{tabular}

$a$ : From two-dimensional Gaussian fitting; "size" is deconvolved source size. If no size is given, the source could not be deconvolved from the beam, and thus its appearance cannot be distinguished from that of a point source. Statistical uncertainties are indicated by the number of significant figures.

Table 3. Properties of spectral lines used in kinematic analysis.

\begin{tabular}{|c|c|c|c|c|c|}
\hline Species & Transition & $\begin{array}{c}\text { Frequency } \\
(\mathrm{GHz})\end{array}$ & $\begin{array}{c}\mathrm{E}_{\text {upper }} \\
(\mathrm{K})\end{array}$ & Catalog $^{a, b}$ & Notes $^{c}$ \\
\hline $\mathrm{CH}_{3} \mathrm{CN}$ & $J=12-11, K=8$ & 220.47581 & 525.6 & JPL & \\
\hline $\mathrm{HC}_{3} \mathrm{~N} \mathrm{v}_{7}=1$ & $J=24-23 \mathrm{l}=1 \mathrm{f}$ & 219.17376 & 452.3 & CDMS & \\
\hline $\mathrm{HC}_{3} \mathrm{~N}_{7}=1$ & $J=24-23 \mathrm{l}=1 \mathrm{e}$ & 218.86080 & 452.1 & CDMS & \\
\hline $\mathrm{CH}_{3} \mathrm{OH}(A)$ & $18_{3,16}-17_{4,13}$ & 232.78350 & 446.5 & CDMS & \\
\hline $\mathrm{CH}_{3} \mathrm{CN}$ & $J=12-11, K=7$ & 220.53932 & 418.6 & JPL & PA, DT_ALL \\
\hline $\mathrm{CH}_{3} \mathrm{OH}(E)$ & $15_{4,11}-16_{3,13}$ & 229.58907 & 374.4 & CDMS & \\
\hline $\mathrm{CH}_{3} \mathrm{OH} \mathrm{v} \mathrm{v}_{t}=1(A)$ & $6_{1,5}-7_{2,6}$ & 217.29920 & 373.9 & CDMS & PA, DT_ $\mathrm{CH}_{3} \mathrm{OH}$ \\
\hline $\mathrm{CH}_{3} \mathrm{CN}$ & $J=12-11, K=5$ & 220.64108 & 247.4 & JPL & \\
\hline $\mathrm{HNCO}^{d}$ & $10_{2,9}-9_{2,8}$ & 219.73385 & 228.3 & CDMS & \\
\hline $\mathrm{CH}_{3} \mathrm{CN}$ & $J=12-11, K=4$ & 220.67929 & 183.1 & JPL & PA, DT_ALL \\
\hline $\mathrm{CH}_{3} \mathrm{OH}(A)$ & $10_{2,8}-9_{3,7}$ & 232.41859 & 165.4 & CDMS & PA, DT_CH ${ }_{3} \mathrm{OH}$ \\
\hline $\mathrm{CH}_{3} \mathrm{OH}(A)$ & $10_{2,9}-9_{4,6}$ & 231.28110 & 165.3 & CDMS & PA, DT_ $\mathrm{CH}_{3} \mathrm{OH}$ \\
\hline $\mathrm{CH}_{3} \mathrm{CH}_{2} \mathrm{CN}$ & $27_{0,27}-26_{0,26}$ & 231.99041 & 157.7 & CDMS & \\
\hline $\mathrm{CH}_{3} \mathrm{CH}_{2} \mathrm{CN}$ & $26_{2,25}-25_{2,24}$ & 229.26516 & 154.0 & CDMS & \\
\hline $\mathrm{CH}_{3} \mathrm{CH}_{2} \mathrm{CN}$ & $26_{1,25}-25_{1,24}$ & 231.31042 & 153.4 & CDMS & \\
\hline $\mathrm{CH}_{3} \mathrm{CH}_{2} \mathrm{CN}$ & $25_{2,24}-24_{2,23}$ & 220.66092 & 143.0 & CDMS & PA, DT_ALL \\
\hline $\mathrm{CH}_{3} \mathrm{CN}$ & $J=12-11, K=3$ & 220.70902 & 133.2 & JPL & PA, DT_ALL \\
\hline $\mathrm{HC}_{3} \mathrm{~N}$ & $J=24-23$ & 218.32472 & 131.0 & CDMS & \\
\hline $\mathrm{CH}_{3} \mathrm{OCHO}(A)^{e}$ & $20_{1,20}-19_{1,19}$ & 216.96590 & 111.5 & JPL & \\
\hline OCS & $J=19-18$ & 231.06099 & 110.9 & CDMS & PA, DT_ALL \\
\hline $\mathrm{HNCO}$ & $10_{1,10}-9_{1,9}$ & 218.98101 & 101.1 & CDMS & PA, DT_ALL \\
\hline $\mathrm{CH}_{3} \mathrm{CN}$ & $J=12-11, K=2$ & 220.73026 & 97.4 & JPL & PA, DT_ALL \\
\hline $\mathrm{CH}_{3} \mathrm{OH}(E)$ & $8_{0,8}-7_{1,6}$ & 220.07849 & 96.6 & CDMS & PA, DT_CH ${ }_{3} \mathrm{OH}$ \\
\hline $\mathrm{CH}_{3} \mathrm{OH}(E)^{f}$ & $8_{-1,8}-7_{0,7}$ & 229.75876 & 89.1 & CDMS & PA, DT_ $\mathrm{CH}_{3} \mathrm{OH}$ \\
\hline $\mathrm{H}_{2} \mathrm{CO}$ & $3_{2,1}-2_{2,0}$ & 218.76007 & 68.1 & CDMS & PA, DT_ALL \\
\hline $\mathrm{HNCO}$ & $10_{0,10}-9_{0,9}$ & 219.79827 & 58.0 & CDMS & \\
\hline $\mathrm{CH}_{3} \mathrm{OH}(E)$ & $5_{1,4}-4_{2,2}$ & 216.94560 & 55.9 & CDMS & \\
\hline $\mathrm{CH}_{3} \mathrm{OH}(E)$ & $3_{-2,2}-4_{-1,4}$ & 230.02706 & 39.8 & CDMS & PA, DT_CH ${ }_{3} \mathrm{OH}$ \\
\hline $\mathrm{SO}$ & $6_{5}-5_{4}$ & 219.94944 & 35.0 & CDMS & \\
\hline $\mathrm{H}_{2} \mathrm{CO}$ & $3_{0,3}-2_{0,2}$ & 218.22219 & 21.0 & CDMS & \\
\hline $\mathrm{DCN}$ & $J=3-2$ & 217.23854 & 20.9 & CDMS & PA, DT_ALL \\
\hline
\end{tabular}

${ }^{a}$ CDMS = http://www.astro.uni-koeln.de/cgi-bin/cdmssearch

$b \mathrm{JPL}=$ http://spec.jpl.nasa.gov/ftp/pub/catalog/catform.html

c PA: line included in calculation of median position angle (Section 3.2); DT_ALL: Potentially 'disc tracing' - results of centroid plot included in the kinematic fitting, all lines excluding $\mathrm{CH}_{3} \mathrm{OH}$ (Section 4.1); DT_ $\mathrm{CH}_{3} \mathrm{OH}$ : Potentially 'disc tracing' — results of centroid plot included in the kinematic fitting, $\mathrm{CH}_{3} \mathrm{OH}$ lines (Section 4.1)

${ }^{d}$ Blended with the HNCO $10_{2,8}-9_{2,7}$ line at $219.73719 \mathrm{GHz}$, which has the same $\mathrm{E}_{\text {upper }}$ and CDMS intensity; the velocity separation from this reference transition is $-4.56 \mathrm{~km} \mathrm{~s}^{-1}$.

$e$ Blended with three other $\mathrm{CH}_{3} \mathrm{OCHO}$ transitions with the same line intensity and $\mathrm{E}_{\text {upper; }}$ the velocity separations from this reference transition are $+1.57,-0.48$, and $-2.10 \mathrm{~km} \mathrm{~s}^{-1}$.

$f$ This line exhibits thermal emission towards MM1, though maser emission is observed towards the G11.92-0.61 outflow lobes (Cyganowski et al. 2011a). 

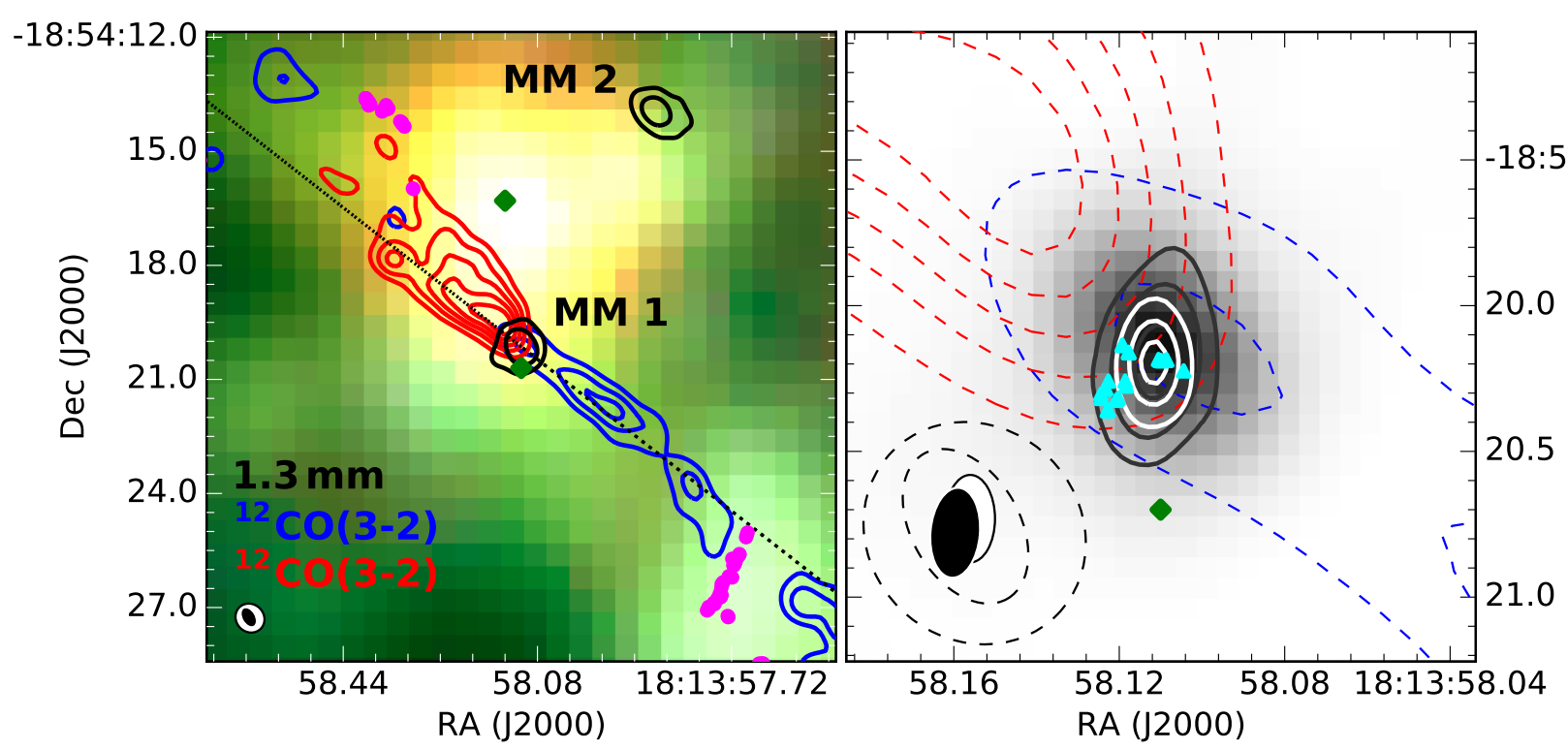

Figure 1. Left: Contours of SMA $1.3 \mathrm{~mm}$ VEX continuum (black) and blue/redshifted ${ }^{12} \mathrm{CO}(3-2)$ emission overlaid on a three-color Spitzer image (RGB: 8.0, 4.5, 3.6 $\mu \mathrm{m}$ ). The outflow position angle $\left(52^{\circ}\right)$ is indicated by the dotted black line (see Section 3.2 ). The field of view is centred on MM1, and MM2 is visible $\sim 7^{\prime \prime}$ to the northwest. Positions of $44 \mathrm{GHz}$ Class I $\mathrm{CH}_{3} \mathrm{OH}$ masers (magenta circles) and $6.7 \mathrm{GHz}$ Class II $\mathrm{CH}_{3} \mathrm{OH}$ masers (green diamonds) from Cyganowski et al. (2009) are marked. The 6.7 GHz maser $\sim 5^{\prime \prime}$ north of MM1 is coincident with the millimetre continuum source MM3 (Cyganowski et al. 2011a, undetected in the VEX observations). Right: Zoomed view of MM1, showing the SMA $1.3 \mathrm{~mm}$ VEX continuum (greyscale, linear from 0 to $0.1 \mathrm{Jy}_{\text {beam }}{ }^{-1}$ ), ${ }^{12} \mathrm{CO}(3-2)$ emission (blue/red dashed contours) and $6.7 \mathrm{GHz}$ maser (diamond). In addition, VLA $3.0 \mathrm{~cm}$ (white) and $0.9 \mathrm{~cm}$ (black) contours are overlaid, along with positions of $22 \mathrm{GHz} \mathrm{H}_{2} \mathrm{O}$ masers (cyan triangles, Moscadelli et al. 2016). Beams are shown at lower left in each panel. Levels: $1.3 \mathrm{~mm}$ : $(5,25) \sigma$, where $\sigma=0.7 \mathrm{mJy}$ beam ${ }^{-1} ; 3.0 \mathrm{~cm}:(5,15,25) \sigma$, where $\sigma=6.1 \mu \mathrm{Jy}_{\text {beam }}{ }^{-1} ; 0.9 \mathrm{~cm}:(5,15,50) \sigma$, where $\sigma=7.6 \mu \mathrm{Jy} \mathrm{beam}^{-1} ;{ }^{12} \mathrm{CO}$ : $0.8 \mathrm{Jy}^{-1} \mathrm{beam}^{-1} \mathrm{~km} \mathrm{~s}^{-1} \times(5,10,15)$ (blue) $\times(5,10,15,20,25)$ (red).

lines from Table 3, chosen to represent the range of chemical species, line emission morphologies, kinematics, and line excitation temperatures present in our SMA VEX $1.3 \mathrm{~mm}$ data. Strikingly, all ten species show a consistent velocity gradient across the MM1 (sub)millimetre continuum source, with a sharp transition from redshifted emission (to the South-East) to blueshifted emission (to the North-West). The orientation of the velocity gradient is consistent with that seen at $\sim 2$ '. 4 resolution by Cyganowski et al. (2011a) in $\mathrm{SO}, \mathrm{HNCO}, \mathrm{CH}_{3} \mathrm{OH}$, and $\mathrm{CH}_{3} \mathrm{CN}$. We measured the position angle of the velocity gradient for each of the 31 transitions in Table 3 by calculating the position angle of the line joining the RA/Dec centroid positions of the emission in the most redshifted and the most blueshifted velocity channels (a similar approach to that of Hunter et al. 2014). The median and standard deviation of the position angle across the fifteen transitions that we select as potentially disc-tracing based on our kinematic fitting and moment analysis (Section 4.1 , Table 3 ) is $127 \pm 18^{\circ}$. We note that the variation in the median position angle from including a different selection of lines in the calculation is well within the scatter indicated by the standard deviation of $18^{\circ}$, and adopt a position angle of $127^{\circ}$ for constructing the position-velocity (PV) diagrams presented in Figure 4.

Notably, the position angle of the velocity gradient is nearly identical to that of the $2 \mathrm{D}$ Gaussian model fit to the $1.3 \mathrm{~mm}$ dust emission of $125 \pm 7^{\circ}$ (Cyganowski et al. 2014). This alignment, and the sharp transition from redshifted to blueshifted emission, are reminiscent of Keplerian discs observed around low-mass stars (e.g. Hughes et al. 2011; Walsh et al. 2014). The position angles of MM1's velocity gradient and dust emission are also nearly perpendicular to that of the high-velocity bipolar molecular outflow driven by MM1. The outflow position angle, estimated as the position angle of the line joining the peaks of the redshifted and blueshifted ${ }^{12} \mathrm{CO}(3-2)$ lobes, is $\sim 52^{\circ}$ (Figure 1 ). For the innermost part of the outflow (shown in the right panel of Figure 1), the estimated position angle is $\sim 45^{\circ}$, an offset of $82^{\circ}$ from the velocity gradient seen in the compact molecular line emission. Both estimates of the outflow position angle are consistent with the outflow being perpendicular to the velocity gradient in the compact gas, within the scatter in our estimate of the latter. This configuration strongly suggests a disc-outflow system, and motivates a more detailed examination of the kinematics of MM1 by means of PV diagrams (Figure 4).

As illustrated in Figure 4, the MM1 PV diagrams exhibit the characteristic pattern expected for a Keplerian disc - the highest velocities are seen closest to the central source, while the most spatially extended emission is seen at lower velocities (e.g. closer to the $\mathrm{V}_{\mathrm{LSR}}$ of the system). These key features are consistent across a range of molecular tracers and transition excitation energies, and support our use of Keplerian disc models to interpret the kinematics of MM1 (Section 4.1). There are also, however, some potentially illuminating differences amongst the prospective disc 


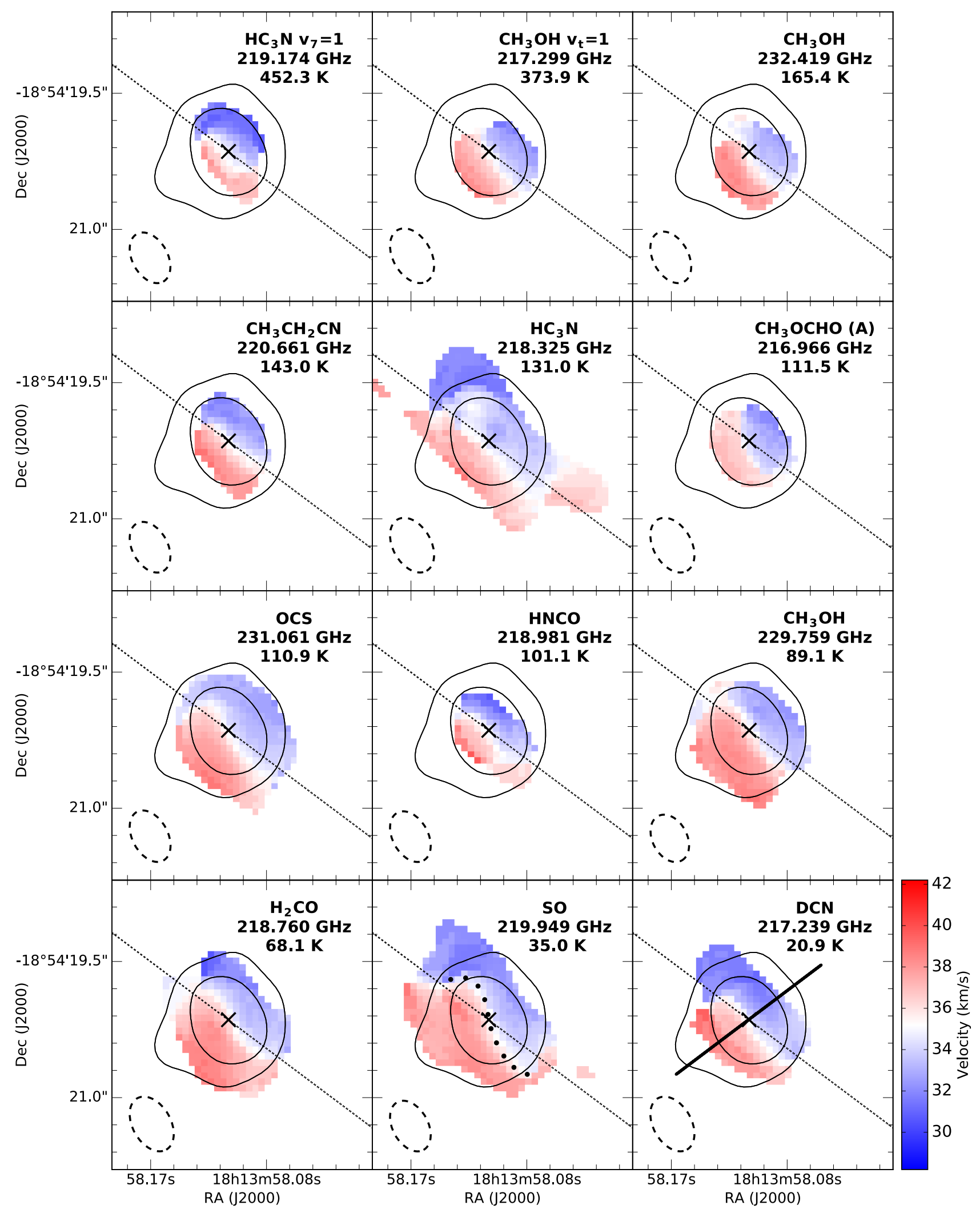

Figure 2. First moment maps (colourscale) of selected spectral lines from Table 3, overlaid with contours of the SMA VEX 1.3 mm continuum emission (levels $5 \& 25 \sigma$, where $\sigma=0.7 \mathrm{mJy}_{\text {beam }}^{-1}$ ). The colourscale is centred on the systemic velocity of the system, $35.2 \mathrm{~km} \mathrm{~s}^{-1}$ (Cyganowski et al. 2011a). Each panel is labeled with the species name and the excitation energy (in Kelvin) of the upper level of the transition. The position angle of the outflow from Fig 1 is shown with a dashed black line. Black dots in the lower middle panel denote the twist in the zero-velocity gas (Section 3.2). The cut used to generate the PV diagrams in Figure 4 is shown in the lower right panel with a solid black line. The beam is shown in the lower left of each panel as a dashed ellipse. 


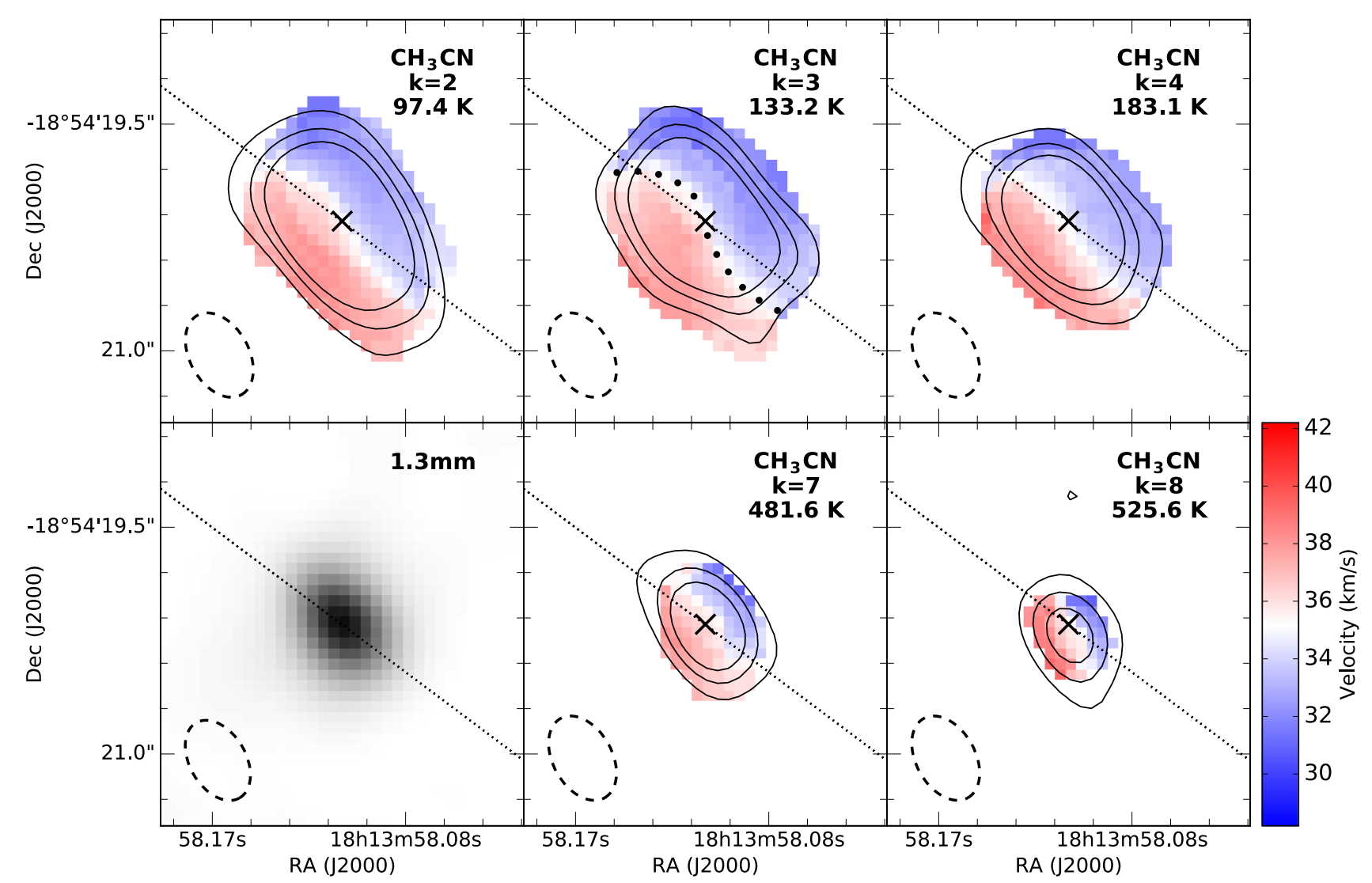

Figure 3. First moment maps for minimally-blended $\mathrm{CH}_{3} \mathrm{CN}$ transitions (we omit $K=6$ as it is blended with $\mathrm{HNCO}$ and $K=5$ as it is blended with $\mathrm{CH}_{3}^{13} \mathrm{CN} K=0$ ), overlaid with zeroth moment contours and displayed alongside the 1.3 mm continuum emission from Figure 1. The excitation energy (in Kelvin) of the upper level of each transition is indicated. Black dots in the upper middle panel denote the twist in the zero-velocity gas (Section 3.2). The continuum peak is marked with a black cross, and the beam is shown in the lower left corner of each panel as a dashed ellipse. Contours are 3,6 and $9 \sigma$, where $\sigma$ for the zeroth moment maps is $0.19,0.22,0.19,0.16$ and $0.16 \mathrm{Jy}_{\text {beam }}{ }^{-1}$, respectively.

tracers. Notably, most of the lines do not peak towards the central source, instead showing offsets in position and/or velocity (Figure 4: the position of the $1.3 \mathrm{~mm}$ continuum peak from our SMA VEX observations is defined as offset=0' 0 ). More surprisingly, these offsets do not appear to be simple functions of molecular abundance or transition excitation energy, in contrast to the pattern seen in the candidate massive disc NGC 6334I(N)-SMA 1b (Hunter et al. 2014). In MM1, low-temperature transitions of abundant molecules, including $\mathrm{DCN}(3-2)$ and $\mathrm{H}_{2} \mathrm{CO}\left(3_{2,1}-2_{2,0}\right)$, show a doublepeaked structure with a local minimum towards the central source, consistent with a radial temperature gradient (increasing inwards, see also Section 3.3) and a moderately edge-on viewing angle. However, higher-temperature transitions of $\mathrm{CH}_{3} \mathrm{CN}$ and $\mathrm{CH}_{3} \mathrm{OH}$ also show notably doublepeaked or asymmetric structure. In contrast, the peak of the OCS(19-18) emission (with a moderate $\mathrm{E}_{\text {upper }}$ of $110.9 \mathrm{~K}$ ) is coincident with the central source. Interestingly, $\mathrm{CH}_{3} \mathrm{CN}$ emission in two other candidate discs around (proto)O-stars $\left(\mathrm{CH}_{3} \mathrm{CN} K=3\right.$ in NGC $6334 \mathrm{I}(\mathrm{N})-\mathrm{SMA} 1 \mathrm{~b}$ and $K=2, K=4$, and $K=6$ in AFGL4176-mm1: Hunter et al. 2014; Johnston et al. 2015, respectively) exhibits asymmetries similar to those we observe in $\mathrm{CH}_{3} \mathrm{CN}$ towards MM1, though these asymmetries are not reproduced by current models (e.g. Figure 4 of Johnston et al. 2015). In MM1, as in NGC 6334I(N)-SMA 1b, HNCO differs from other species in displaying a compact morphology in PV diagrams. Based on the present data, it is difficult to disentangle the effects of molecular abundance (e.g. potential chemical segregation, as observed in AFGL2591 by Jiménez-Serra et al. 2012) from those of molecular excitation in the MM1 disc. Higher angular resolution (sub)millimetre observations would be required to obtain well-resolved images of many different molecular species.

The moment maps shown in Figure 2 also highlight that though all species share a consistent velocity gradient (from redshifted in the South-East to blueshifted in the NorthWest), $\mathrm{SO}\left(6_{5}-5_{4}\right)$ and $\mathrm{HC}_{3} \mathrm{~N} v=0(24-23)$ exhibit emission that is notably spatially extended compared to the millimetre continuum and to other molecules. The SO line is lowtemperature $\left(\mathrm{E}_{\text {upper }}=35.0 \mathrm{~K}\right)$ and is clearly detected in the G11.92-0.61 outflow in our lower-resolution SMA data (e.g. Figure 3 of Cyganowski et al. 2011a), suggesting possible outflow contribution to the emission detected in our highresolution SMA VEX observations. The extended emission from $\mathrm{HC}_{3} \mathrm{~N} v=0(24-23)$, with its higher excitation energy $\left(E_{\text {upper }}=131.0 \mathrm{~K}\right)$, is perhaps more surprising, though recent observations have found that $\mathrm{HC}_{3} \mathrm{~N}$ emission is as- 


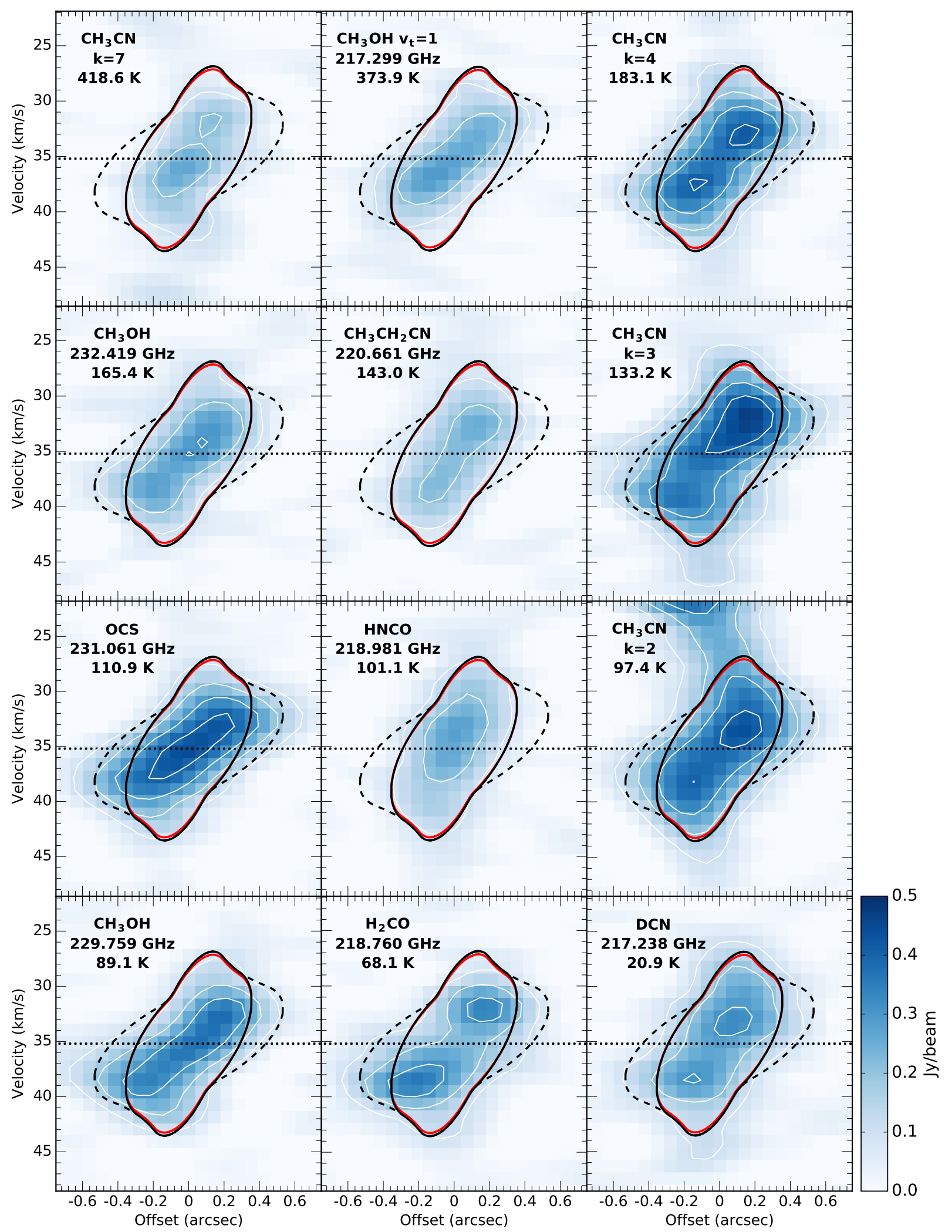

Figure 4. Position-velocity diagrams of selected lines from Table 3, ordered by decreasing $\mathrm{E}_{\text {upper }}$. Solid white contours correspond to levels of $0.2,0.3,0.4$ and $0.5 \mathrm{Jy}_{\text {beam }}^{-1}$. The $\mathrm{v}_{\mathrm{lsr}}$ of the system from Cyganowski et al. (2011a), $35.2 \mathrm{~km} \mathrm{~s}^{-1}$, is shown with a horizontal dotted line; the position of the $1.3 \mathrm{~mm}$ continuum peak from the SMA VEX observations is defined as offset=0' 0 . The $\mathrm{CH}_{3} \mathrm{CN} K=2$ transition displays contamination at high blueshifted velocities from a nearby line. Overlaid are the best fitting disc models from Section 4.1: the best fit model for all lines, excluding $\mathrm{CH}_{3} \mathrm{OH}\left(\mathrm{M}_{\mathrm{enc}}=60 \mathrm{M}_{\odot}, i=35^{\circ}, \mathrm{R}_{\mathrm{o}}=1200 \mathrm{au}\right)$ is shown with a solid black line, and the best fit model for $\mathrm{CH}_{3} \mathrm{OH}$ lines only $\left(\mathrm{M}_{\mathrm{enc}}=34 \mathrm{M}_{\odot}, i=52^{\circ}, \mathrm{R}_{\mathrm{o}}=1200 \mathrm{au}\right)$ is shown with a solid red line. These two models are almost identical in position-velocity space. The dashed black line shows a model identical to that of the best fitting disc model for all lines (excluding $\mathrm{CH}_{3} \mathrm{OH}$ ), but with $\mathrm{R}_{\mathrm{o}}=1800 \mathrm{au}$, providing a better fit to some transitions. 
sociated with gas shocked by outflows driven by low-mass (proto)stars (e.g. Shimajiri et al. 2015; Benedettini et al. 2013). Within $\sim 10 \mathrm{~km} \mathrm{~s}^{-1}$ of the systemic velocity (e.g., the range shown in Figure 2), the kinematics of outflow-tracing molecules such as ${ }^{12} \mathrm{CO}$ and $\mathrm{HCO}^{+}$are very confused, with both redshifted and blueshifted emission detected to both the NE and SW of MM1 (e.g. Figures 7 and 8 of Cyganowski et al. 2011a). It is thus plausible that (the base of) the MM1 outflow contributes to the $\mathrm{SO}$ and $\mathrm{HC}_{3} \mathrm{~N}$ emission detected in our SMA VEX observations. In addition, given the apparent elongation along the outflow axis in moment 0 maps of many of the lines examined, it is also possible that the base of the outflow contributes to more lines, though we note that as the beam is elongated in a similar direction, this effect is difficult to quantify with our current observations.

Several of the moment 1 maps (e.g. $\mathrm{CH}_{3} \mathrm{CN} K=2,3,4$, $\operatorname{OCS}(19-18), \mathrm{H}_{2} \mathrm{CO}\left(3_{2,1}-2_{2,0}\right), \mathrm{SO}\left(6_{5}-5_{4}\right), \mathrm{OCS}(19-18)$ and $\mathrm{DCN}(3-2))$ also exhibit an interestingly asymmetric feature: a twisted structure that is most clearly seen by examining the gas moving at the systemic velocity (white in Figures 2 $\& 3$, marked with black dots). Based on the orientation of the blue- and red-shifted emission (i.e. in opposition to the movement of the outflowing material), we suggest that this twisting is likely due to the effect of infalling material. Such an interpretation is also strengthened by the requirement of an infall component to be used in our modelling of the PV diagrams (see Figure 4 and Section 4.1)

\subsection{Extent of gas and dust emission in the disc}

The thermal dust emission from MM1 is unresolved in our SMA $1.3 \mathrm{~mm}$ VEX observations, with a fitted deconvolved source size of $1150 \times 960$ au (Cyganowski et al. 2014). In contrast, the molecular line emission from the disc is marginally resolved in some lower-excitation transitions. For example, for the blended $K=0$ and $K=1$ components of $\mathrm{CH}_{3} \mathrm{CN}(12-$ 11 ), the deconvolved size of the $2 \mathrm{D}$ Gaussian fit to the moment 0 map is $0{ }^{\prime} 80 \pm 00^{\prime} 06 \times 00^{\prime} 53 \pm 00^{\prime} 04$ (P.A. $=47 \pm 8$ ), or $\sim 2700 \times 1800 \mathrm{au}$. The $\mathrm{CH}_{3} \mathrm{CN}$ ladder is of particular interest, as the relative spatial extents of the different k-components are expected to depend primarily on gas temperature. Figure 3 presents contours of integrated intensity for the $K=2$, $K=3, K=4, K=7$, and $K=8$ transitions of $\mathrm{CH}_{3} \mathrm{CN}(12-11)$, which range in excitation energy from $\mathrm{E}_{\text {upper }}=97.4-525.6 \mathrm{~K}$, overlaid on the moment 1 maps of these transitions (we omit $K=0$ and $K=1$ because they are blended with each other, $K=6$ because it is blended with $\operatorname{HNCO}\left(10_{1,9}-9_{1,8}\right.$ at 220.585 $\mathrm{GHz}$, and $K=5$ because it is blended with $\mathrm{CH}_{3}^{13} \mathrm{CN} \mathrm{K}=0$ ). As illustrated by Figure 3, all transitions display a similar velocity gradient, but the low- $k$, low-excitation transitions of $\mathrm{CH}_{3} \mathrm{CN}$ are significantly more extended than the high- $k$, high-excitation transitions. This pattern of decreasing spatial extent with increasing excitation energy is suggestive of an increasing temperature gradient towards a central source. The temperature structure of the gas around MM1, as determined from modelling the $\mathrm{CH}_{3} \mathrm{CN}$ emission, is discussed further in Section 4.2.

To investigate the differing extents of the dust and gas emission, we performed simple azimuthal averages (i.e. not accounting for any ellipicity effects) on both the continuum and the $\mathrm{CH}_{3} \mathrm{CN} K=3$ line emission (the lowest-energy k

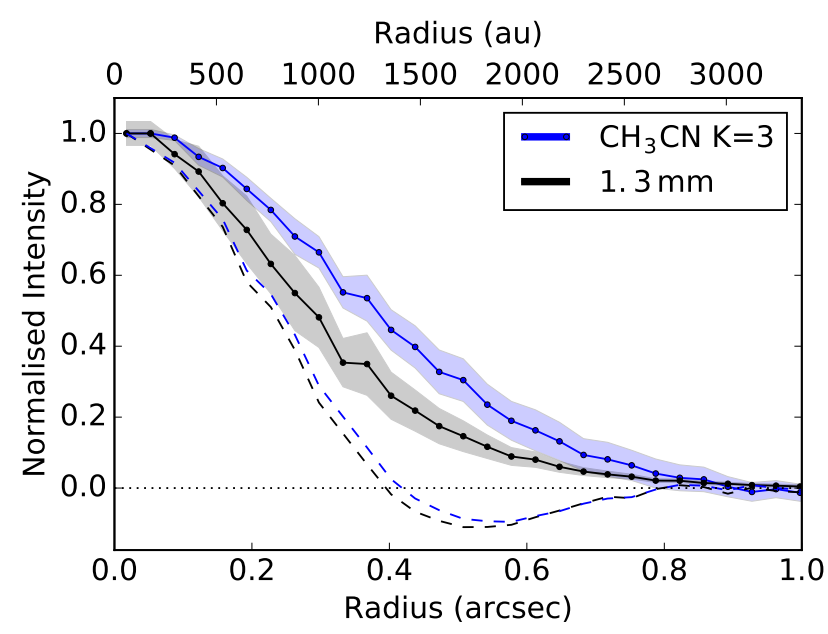

Figure 5. Azimuthal averages of the normalised intensity of the $\mathrm{CH}_{3} \mathrm{CN} \mathrm{K}=3$ line (blue) and $1.3 \mathrm{~mm}$ continuum emission (black), centered around the bi-section between the most red and blue shifted channel centroids, with $1 \sigma$ errors shown as shaded regions. Azimuthal averages of the beams are shown with dashed lines.

component that is well separated from neighboring lines, see also top panel of Figure 7). As shown in Figure 5, this analysis confirms that the $\mathrm{CH}_{3} \mathrm{CN} K=3$ emission is more extended than the $1.3 \mathrm{~mm}$ continuum, by approximately $0 . ' 1$ at maximum deviation. Figure 5 also highlights that the continuum emission, unlike the $K=3$ line emission, is unresolved by our SMA observations. A larger spatial extent of gas emission in comparison to dust continuum emission is a result often seen in observations of circumstellar discs around lower-mass young stars (e.g. Guilloteau et al. 2011; Pérez et al. 2012; de Gregorio-Monsalvo et al. 2013). In such systems, the spatial difference is often suggested to be due to either radial migration of the dust towards the central star, or viscous outward spreading of the gas (e.g. Alexander et al. 2006).

\section{DISCUSSION}

\subsection{Molecular line modelling: gas kinematics}

In order to further investigate the kinematic origin of each emission line, we fitted a 2-dimensional Gaussian to each channel using the CASA IMFIT routine. For a given line and channel, this fit provides the location of the centroid, whose position on the sky is recorded along with the channel velocity. This process is then repeated for all channels across a given line. The morphology of the resulting centroid plots often exhibits a closed loop structure, with the most red- and blue-shifted channels closest together, and velocities between these channels tracing an arc across the sky (Figure 6). However, the size of this loop varies from line to line, with some lines exhibiting more linear centroid plots (e.g. the $\mathrm{CH}_{3} \mathrm{OH}$ transitions). For each line, we define a velocity centre - the on-sky position that bisects the positions of the most redand blue-shifted channel centroids. The position of this velocity centre for each line differed slightly, however all were 
consistent to within approximately $0{ }^{\prime} 05$ (or $\sim 200$ au at the distance of MM1).

Based on careful examination of the individual line centroid plots and the corresponding moment 1 maps, we selected a subset of potentially disc-tracing (DT) lines. In order to model the kinematic origin of these lines, we adopted an approach similar to that presented in Sánchez-Monge et al. (2013), and use a model of a geometrically thin Keplerian disc (Maret 2015) to compare with the centroid information. The model disc is characterised by a central mass $\left(M_{\star}\right)$, an outer radius $\left(R_{o}\right)$ and an inclination to the line of sight ( $i$, where $i=0$ corresponds to a face on disc), and is assumed to emit uniformly as a function of radius (though we note that adopting different intensity distributions does not alter the results of our fitting). The model is given a number of velocity channels with width consistent with that of our observations, and is assumed to lie at the same distance as MM1 (3.37 kpc).

For each combination of free parameters, a spectral cube is created for the model, and then a first moment map is produced. This is then compared to the centroid plots. The chi-squared landscape is explored in a grid based fashion using the SCIPY OPTIMIZE BRUTE module, where the ranges for each parameter were $1<\mathrm{M}_{\star}<90 \mathrm{M}_{\odot}, 600<\mathrm{R}_{\mathrm{o}}<1400 \mathrm{au}$, and $0<i<90^{\circ}$. Once a minimum within the grid is recovered, a Nelder-Mead (or AMEOBA) minimisation technique is used to refine the best fitting parameters. Due to the slightly different morphologies of the $\mathrm{CH}_{3} \mathrm{OH}$ centroid plots, we chose to perform the fitting on two collections of centroid locations - those obtained from all lines but excluding $\mathrm{CH}_{3} \mathrm{OH}$ (labelled 'DT_ALL' in Table 3), and only the $\mathrm{CH}_{3} \mathrm{OH}$ lines (labelled 'DT_ $\mathrm{CH}_{3} \mathrm{OH}$ ' in Table 3 ). The best fitting disc model for all lines surrounds an enclosed mass of $60_{-27}^{+21} \mathrm{M}_{\odot}$ at an inclination of $35_{-6}^{+20 \circ}$. The best fitting disc model for only the $\mathrm{CH}_{3} \mathrm{OH}$ transitions surrounds an enclosed mass of $34_{-12}^{+28} \mathrm{M}_{\odot}$ at an inclination of $52_{-14}^{+11 \circ}$. In both cases, the disc extended to $1200 \mathrm{au}$. Figure 6 shows the resulting best fitting models for each of these collections.

Based on the results of the fitting to the centroid maps, we can follow the prescription of Cesaroni et al. (2011) and overlay theoretical position-velocity models for these Keplerian discs on Figure 4. In this case, the region on a positionvelocity diagram within which emission is expected can be expressed as

$$
V=\sqrt{G M} \frac{x}{R^{\frac{3}{2}}}+\sqrt{2 G M} \frac{z}{R^{\frac{3}{2}}}
$$

where the first term is the contribution from the Keplerian disc, and the second the contribution due to free fall. $V$ is the velocity component along the line of sight, $M$ is the enclosed mass, $x$ and $z$ are the co-ordinates along the disc plane and line of sight, respectively, and $R=\sqrt{x^{2}+z^{2}}$ is the distance from the centre of the disc.

The models overlaid on Figure 4 correspond to the best fitting solutions found from the centroid fitting - the best fit model for all 'disc-tracing' lines other than $\mathrm{CH}_{3} \mathrm{OH}\left(\mathrm{M}_{\mathrm{enc}}\right.$ $=60 \mathrm{M}_{\odot}, i=35^{\circ}, \mathrm{R}_{\mathrm{o}}=1200 \mathrm{au}$, based on nine transitions) is shown with a solid black line, and the best fit model for 'disc-tracing' $\mathrm{CH}_{3} \mathrm{OH}$ lines $\left(\mathrm{M}_{\mathrm{enc}}=34 \mathrm{M}_{\odot}, i=52^{\circ}, \mathrm{R}_{\mathrm{o}}=\right.$ $1200 \mathrm{au}$, based on six transitions) is shown with a solid red line. These models produce almost identical projections in position-velocity space. For all transitions, the model accurately reproduces the range of velocities displayed in the data. The major differences arise in the spatial extent of the emission, with molecules such as $\mathrm{CH}_{3} \mathrm{CH}_{2} \mathrm{CN}$, HNCO, DCN and $\mathrm{CH}_{3} \mathrm{CN} K=7$ appearing to originate from smaller radii than molecules such as OCS or the lower $K$ transitions of $\mathrm{CH}_{3} \mathrm{CN}$. For this reason, we also overplot a model identical to the best fitting disc model for all lines (excluding $\mathrm{CH}_{3} \mathrm{OH}$ ), but with $\mathrm{R}_{\mathrm{o}}=1800 \mathrm{au}$, as a dashed black line. Interestingly, while there seems to be a dependence of location/extent of emission on excitation energy for the $\mathrm{CH}_{3} \mathrm{CN}$ transitions, there appears not to be a similar trend across all molecules. This can most easily be seen by comparing DCN $\left(\mathrm{E}_{\text {upper }}=20.9 \mathrm{~K}\right)$ with, for example, $\mathrm{CH}_{3} \mathrm{OH} v_{t}=1$ $\left(\mathrm{E}_{\text {upper }}=373.9 \mathrm{~K}\right)$, whose PV diagrams show similar spatial extents.

We can also estimate an inclination angle from the observed ellipicity of the deconvolved Gaussian model for the SMA VEX $1.3 \mathrm{~mm}$ continuum emission (Cyganowski et al. 2014). Assuming circular symmetry, this implies an inclination angle for the dust disc of $\sim 31_{-7^{\circ}}^{+5^{\circ}}$. While we interpret this result with some caution because the continuum is unresolved by our observations, this estimate provides an independent line of evidence for a moderately inclined disc.

\subsection{Molecular line modelling: gas temperatures and physical properties from $\mathrm{CH}_{3} \mathrm{CN}$}

To determine the physical properties of the gas around MM1, we follow the approach of Hunter et al. (2014) and use the CASSIS package to model the line intensities and profiles of the $\mathrm{CH}_{3} \mathrm{CN}$ and $\mathrm{CH}_{3}^{13} \mathrm{CN}$ emission line ladders, which have been shown to provide robust measurements of gas physical conditions in hot cores (e.g. Pankonin et al. 2001; Araya et al. 2005).

In order to quantify any spatial variations in these physical conditions, we extract the $\mathrm{CH}_{3} \mathrm{CN}$ spectra on a pixel-bypixel basis across the location of MM1. The physical model used to fit the data was assumed to be in local thermodynamic equilibrium (LTE), and possessed six free parameters - $\mathrm{CH}_{3} \mathrm{CN}$ column density, excitation temperature, line width, emission region diameter, velocity, and the isotopic ratio of ${ }^{12} \mathrm{C} /{ }^{13} \mathrm{C}$. The ranges explored for each parameter were as follows - column density: $10^{16}<N_{\mathrm{CH}_{3} \mathrm{CN}} \leq$ $10^{18} \mathrm{~cm}^{-2}$; excitation temperature: $40<T_{\text {ex }} \leq 250 \mathrm{~K}$; line width: $3<\Delta v \leq 8 \mathrm{~km} \mathrm{~s}^{-1}$; size: $0.1^{\prime \prime}<\theta \leq 0.5^{\prime \prime}$; velocity: $31<v<42 \mathrm{~km} \mathrm{~s}^{-1}$; isotopic ratio: $55<{ }^{12} \mathrm{C} /{ }^{13} \mathrm{C} \leq 85$. The fitting was performed using a Markov-Chain Monte Carlo (MCMC) minimisation strategy — an initial guess for each parameter is taken, and randomised steps in each parameter are taken to explore the resulting goodness of fit. These steps are initially large, but decrease as the fitting procedure progresses in order to refine the best fitting parameters. The number of iterations was set to 5000, and the cutoff parameter to determine when the step size becomes fixed was set to 2500. The best fitting values for each pixel are taken from the execution that achieved an acceptance rate of 0.5. To ensure only data with a reliable signal-to-noise ratio were used in the fitting procedure, only pixels with spectra where the $K=3$ transition was detected above $5 \sigma$ were included in the modelling, which ensured the fitting routine was al- 

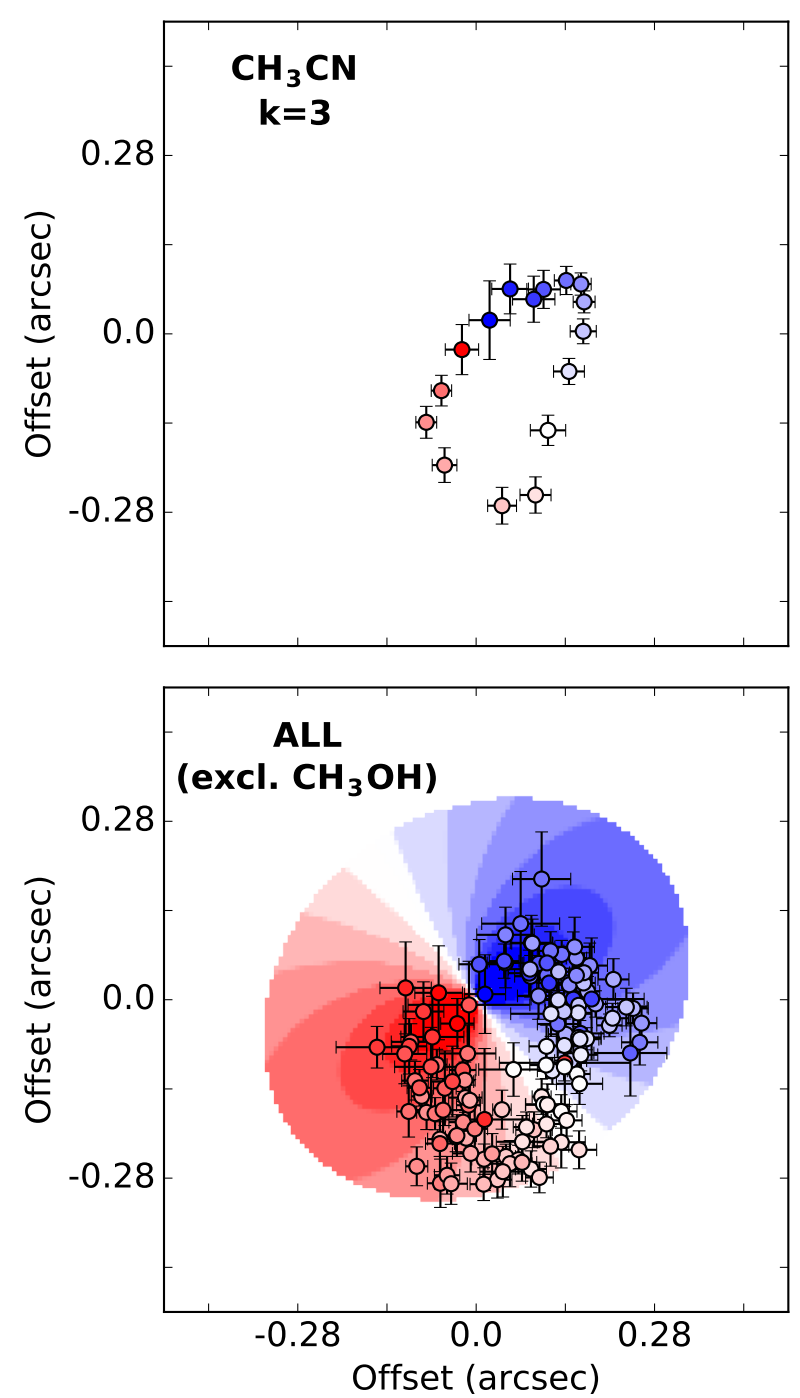
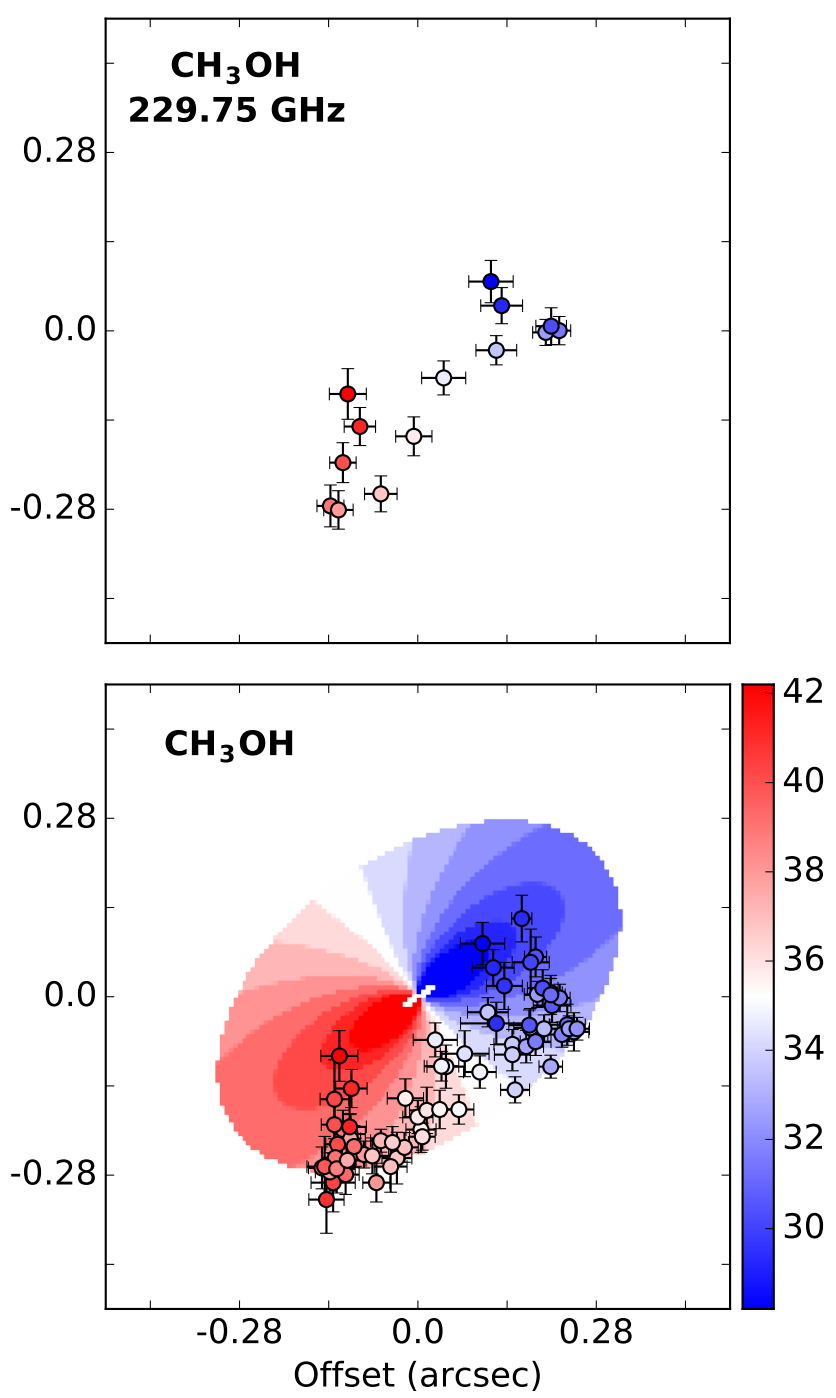

Figure 6. Top: centroid plots for the $\mathrm{CH}_{3} \mathrm{CN} \mathrm{K}=3$ (left) and the $\mathrm{CH}_{3} \mathrm{OH} 229.75 \mathrm{GHz}$ (right) transitions, displaying the differing morphology characteristic of $\mathrm{CH}_{3} \mathrm{OH}$. Bottom: Results of the kinematic fitting procedure described in Section 4.1 for the two collections of centroid points - all 'disc-tracing' lines other than $\mathrm{CH}_{3} \mathrm{OH}$ (left) and 'disc-tracing' $\mathrm{CH}_{3} \mathrm{OH}$ transitions only (right). The best fitting disc model for all lines (except $\mathrm{CH}_{3} \mathrm{OH}$ ) surrounded an enclosed mass of $60_{-27}^{+21} \mathrm{M}_{\odot}$ at an inclination of $35_{-6}^{+20 \circ}$, with an outer radius $\mathrm{R}_{\mathrm{o}}=1200_{-100}^{+100}$ au. The best fitting disc model for only the $\mathrm{CH}_{3} \mathrm{OH}$ transitions surrounded an enclosed mass of $34_{-12}^{+28} \mathrm{M}_{\odot}$ at an inclination of $52_{-14}^{+11 \circ}$, with an outer radius $R_{o}=1200_{-100}^{+100}$ au. In both cases, the position angle of the disc was fixed to $127^{\circ}$. Axes are in offsets with respect to the velocity centre as defined in Section 4.1.

ways given at least the $K=0 / 1$ (blended with each other), $K=2$ and $K=3$ transitions to fit. The subsequent best fitting model spectra were then examined by eye, and any inadequate fits were discarded from the final results.

In all cases, we found that models with a single component of emitting material could not adequately reproduce the line ratios observed in the $\mathrm{CH}_{3} \mathrm{CN}$ and $\mathrm{CH}_{3}^{13} \mathrm{CN}$ spectra. Instead, two components of emitting material were required to adequately reproduce the emission (similar to observations of hot cores, e.g. Cyganowski et al. 2011a; HernándezHernández et al. 2014). In these two component fits, each component was given a set of independent parameters as listed above.

Figure 7 shows the results of the fitting procedure.
The top panel shows the results of a fit to an individual pixel, with the respective transitions of $\mathrm{CH}_{3} \mathrm{CN}$ and $\mathrm{CH}_{3}^{13} \mathrm{CN}$ labelled, along with the transition of ${ }^{13} \mathrm{CO}$ that appears within that range of frequencies. There is good agreement between the line ratios and line widths of the model and the data, suggesting we are placing strong constraints on the parameters. The lower panels of Figure 7 show the results for the first component (left) and second component (right). The first component is characterised by cooler material $(\sim 150 \mathrm{~K})$, with a higher column $\left(\sim 10^{18} \mathrm{~cm}^{-2}\right)$ and a larger linewidth $\left(\sim 8 \mathrm{~km} \mathrm{~s}^{-1}\right)$, while the second component is warmer $(\sim 250 \mathrm{~K})$ with a lower column $\left(\sim 10^{16} \mathrm{~cm}^{-2}\right)$ and exhibits a range of linewidths $\left(5-8 \mathrm{~km} \mathrm{~s}^{-1}\right)$. These two temperature components may be identified with two distinct reservoirs of $\mathrm{CH}_{3} \mathrm{CN}$ in the disc, as seen in recent chemi- 

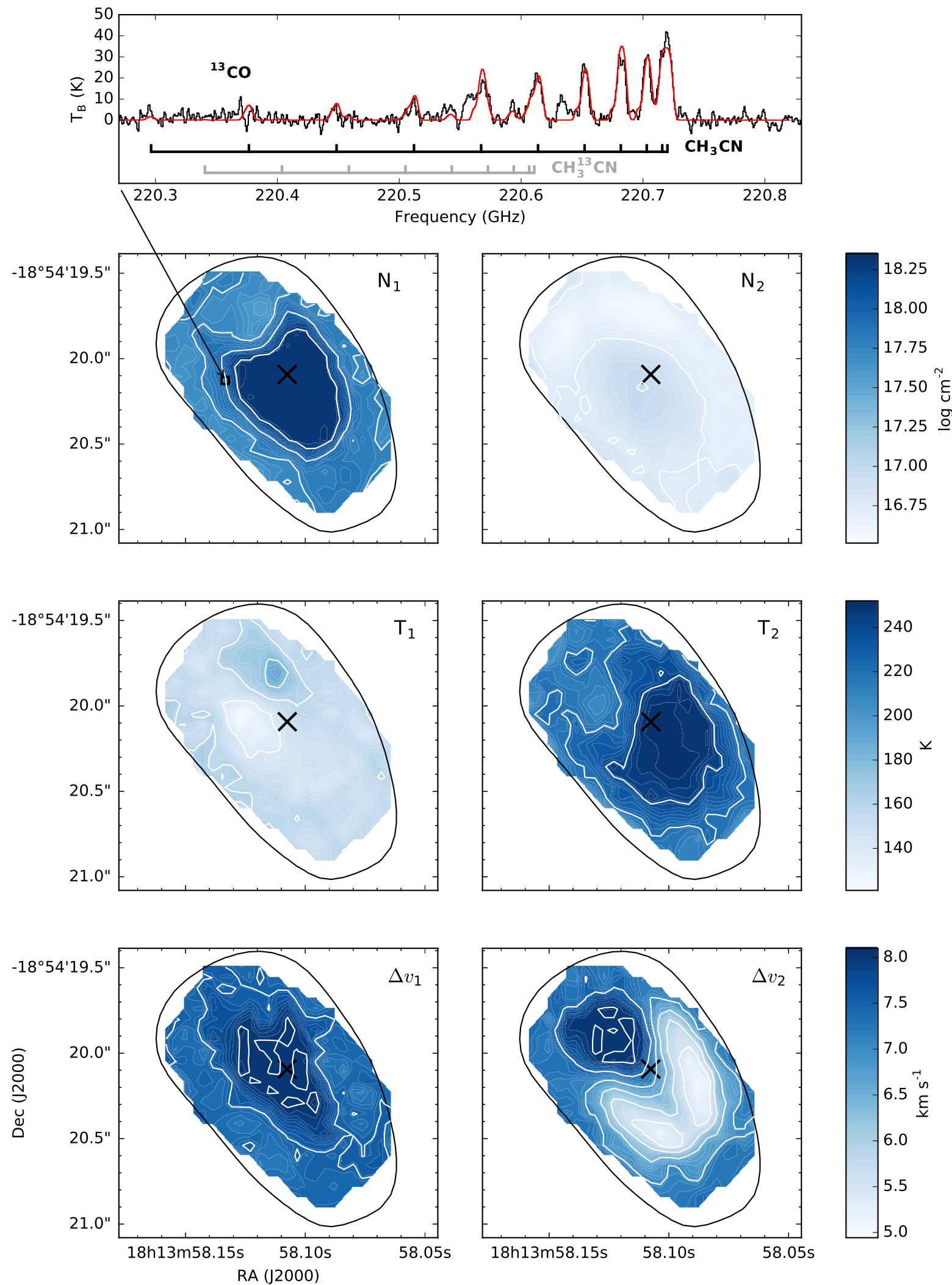

Figure 7. Results from CASSIS fitting to the $\mathrm{CH}_{3} \mathrm{CN}$ spectra across the region of MM1. Top: fitting results (red) to the spectrum (black) from a single pixel. Bottom: Column densities $(N)$, temperatures $(T)$ and line widths $(\Delta v)$ as derived from the fits for the first component (left) and second component (right). White contours correspond to the labeled values with tick marks on each colourbar. The black contour shows the $3 \sigma$ level of the moment 0 map for the $K=2$ transition (where $\sigma=0.19 \mathrm{Jy} \mathrm{beam}^{-1}$ ), and the black cross marks the location of the $1.3 \mathrm{~mm}$ continuum peak. 
Table 4. Measured continuum flux densities included in the spectral energy distribution shown in Figure 8.

\begin{tabular}{lccc}
\hline Wavelength & $\begin{array}{c}\text { Frequency } \\
(\mathrm{GHz})\end{array}$ & $\begin{array}{c}\text { Flux Density } \\
(\mathrm{mJy})\end{array}$ & Reference $^{a}$ \\
\hline $5.5 \mathrm{~cm}$ & 5.50 & $0.075 \pm 0.008$ & M16 \\
$4.8 \mathrm{~cm}$ & 6.2 & $0.112 \pm 0.011$ & M16 \\
$4.3 \mathrm{~cm}$ & 6.98 & $0.086 \pm 0.009$ & M16 \\
$3.0 \mathrm{~cm}$ & 10.0 & $0.161 \pm 0.014$ & This work \\
$2.3 \mathrm{~cm}$ & 13.1 & $0.19 \pm 0.02$ & M16 \\
$1.4 \mathrm{~cm}$ & 21.7 & $0.42 \pm 0.05$ & M16 \\
$1.2 \mathrm{~cm}$ & 24.9 & $0.654 \pm 0.082$ & C14 \\
$0.9 \mathrm{~cm}$ & 33.0 & $0.715 \pm 0.023$ & This work \\
$1.4 \mathrm{~mm}$ & 216.5 & $169 \pm 13$ & C11 \\
$1.3 \mathrm{~mm}$ & 225.1 & $148 \pm 3$ & C14 \\
$1.1 \mathrm{~mm}$ & 273.7 & $397 \pm 6$ & C14 \\
$0.88 \mathrm{~mm}$ & 341.6 & $525 \pm 8$ & C14 \\
\hline
\end{tabular}

$a$ : M16: Moscadelli et al. (2016); C14: Cyganowski et al. (2014); C11: Cyganowski et al. (2011a)

cal models of circumstellar discs around low to intermediate mass young stars. These models show that substantial reservoirs of $\mathrm{CH}_{3} \mathrm{CN}$ can exist in both the disc midplane and disc atmosphere (Walsh et al. 2015). In the upper regions of these disc models, ion-molecule chemistry dominates the production of $\mathrm{CH}_{3} \mathrm{CN}$ (with a small contribution from the thermal desorption of ice mantles), and strong UV fluxes (when present) dominate the destruction through photodissociation. For the midplane regions, which hold the majority of the $\mathrm{CH}_{3} \mathrm{CN}$ reservoir, production is dominated by thermal desorption from ice mantles in regions where the temperature exceeds $150 \mathrm{~K}$, and the molecule simply freezes out in regions with lower temperatures (C. Walsh, private communication). It is interesting to note that while the parameters of the first, lower-temperature component appear relatively symmetric about the continuum peak, the temperature and linewidth of the second component exhibit significant asymmetries towards the South-West. In particular, the high temperatures seen towards the South-West would be consistent with a disc oriented as modelled in Section 4.1, if the disc was sufficiently flared and the disc was optically thick. Such a geometry would also explain the orientation of the looped centroids of emission seen in Section 4.1.

\subsection{Nature of the central cm-wavelength source}

In order to constrain the properties of the central cmwavelength source, we model the free-free and dust emission simultaneously, using an approach similar to that of Hunter et al. (2014). We first construct the spectral energy distribution (SED) of MM1 shown in Figure 8 (filled points) from our new VLA observations, the four datapoints in Cyganowski et al. (2014) $(1.2 \mathrm{~cm}, 1.3 \mathrm{~mm}, 1.1 \mathrm{~mm}$, and $0.88 \mathrm{~mm})$, and the $1.4 \mathrm{~mm}$ CARMA measurement from Cyganowski et al. (2011a). We choose these measurements because they are, by design, as comparable as possible in angular resolution and $u v$-coverage (the new 3.0 and $0.9 \mathrm{~cm}$ VLA data are of higher resolution than the other datasets, but designed to be well-matched to each other). The error bars plotted in Figure 8 represent conservative estimates of the absolute flux calibration uncertainty (15 per cent for SMA $1.3 \mathrm{~mm}$

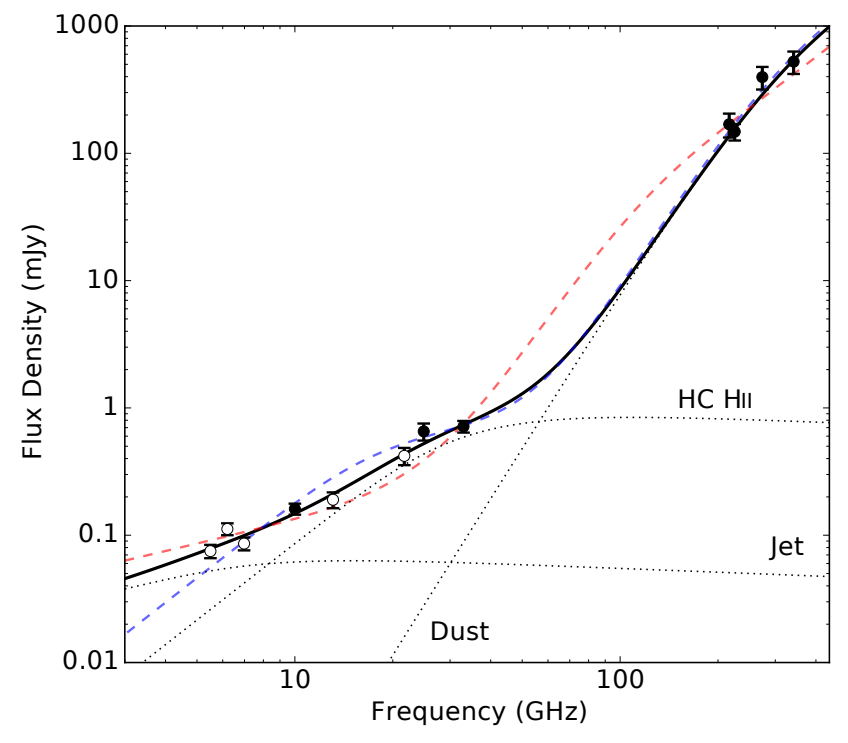

Figure 8. Spectral energy distribution of MM1 from centimetre to submillimetre wavelengths including data from this paper (3.0 and $0.9 \mathrm{~cm}$ VLA), Cyganowski et al. (2014) $(1.2 \mathrm{~cm}$ VLA, $1.3 \mathrm{~mm}, 1.1 \mathrm{~mm}$, and $0.88 \mathrm{~mm}$ SMA), and Cyganowski et al. (2011a) (1.4 mm CARMA), shown as filled points. These measurements are integrated flux densities from two-dimensional Gaussian fitting; the error bars represent the statistical uncertainties from the fit combined in quadrature with conservative estimates for the absolute flux calibration uncertainty (see Section 4.3). Shown as open points are flux densities from Moscadelli et al. (2016) (4.3-5.5, 2.3 and $1.4 \mathrm{~cm}$ VLA, see Table 4). The best fitting model, shown as a solid black line, combines dust emission, a uniform density $\mathrm{HC} \mathrm{H}$ II region, and an ionised jet with a powerlaw density profile (labelled black dotted lines). For comparison, the dashed red line and dashed blue line show a dust and jet model, and a dust and HC H II model, respectively (Section 4.3).

VEX, 5 per cent for VLA $\lambda>2.5 \mathrm{~cm}, 10$ per cent for VLA $\lambda<2.5 \mathrm{~cm}, 20$ per cent for higher-frequency SMA and for CARMA $1.4 \mathrm{~mm}$ ), added in quadrature with the statistical uncertainties from the Gaussian fitting.

To model the centimetre-submillimetre wavelength SED, we combine a free-free model (Olnon 1975) with a single-temperature modified graybody function representing the dust emission (e.g. Gordon 1995; Rathborne et al. 2010). We explored modelling the free-free component with the various Olnon (1975) model geometries for the ionised gas: spherical (uniform density), Gaussian, power law, and truncated power law $\left(n_{e} \propto r^{-2}\right.$, transitioning to a central constant-density sphere to avoid a singularity). We found that the uniform density sphere model best reproduces the centimetre-wavelength shape of the $\operatorname{SED}(\lambda=0.9-3 \mathrm{~cm})$, so we focus on that model for our detailed exploration of parameter space. The initial combined model has seven free parameters: the electron density $\left(\mathrm{n}_{e}\right)$, radius $\left(\mathrm{R}_{e}\right)$, and electron temperature $\left(\mathrm{T}_{e}\right)$ of the ionised sphere and the angular diameter $\left(\theta_{\mathrm{d}}\right)$, temperature $\left(\mathrm{T}_{\mathrm{d}}\right)$, grain opacity index $(\beta)$, and reference opacity $\left(\tau_{1.3 \mathrm{~mm}}\right)$ of the dust emission. Since we have seven data points with which to constrain seven free parameters, we explore parameter space by constructing a 40 by 40 point grid of electron density $\left(10^{5.6}-10^{7.2} \mathrm{~cm}^{-3}\right)$ and 
$\mathrm{R}_{e}$ (15-65 au), and fitting for the other five parameters $\left(\mathrm{n}_{e}\right.$, $\theta_{\mathrm{d}}, \mathrm{T}_{\mathrm{d}}, \beta$, and $\tau_{1.3 \mathrm{~mm}}$ ) at each point in the $\mathrm{n}_{e}-\mathrm{R}_{e}$ grid (see also Hunter et al. 2014). The fit ranges for the free parameters were $\mathrm{T}_{e}=6000-11000 \mathrm{~K}, \beta=0.5-2.5, \theta_{\mathrm{d}}=0.2-2.0^{\prime \prime}$, and $\mathrm{T}_{\mathrm{d}}=150-250 \mathrm{~K} ; \tau_{1.3 \mathrm{~mm}}$ was unconstrained. The range for $\mathrm{T}_{\mathrm{d}}$ was chosen based on our modelling of the $\mathrm{CH}_{3} \mathrm{CN}$ emission (Section 4.2). At high density, as in MM1, gas and dust temperatures are expected to be coupled (e.g. Ceccarelli et al. 1996; Kaufman et al. 1998); since $\beta$ and $\mathrm{T}_{\mathrm{d}}$ are degenerate, we use the gas temperature measurement to better constrain the dust model.

The combined model of a uniform-density ionised sphere and graybody dust emission provides a good fit to the observed centimetre-submillimetre SED of MM1 at wavelengths $\lambda \leq 3 \mathrm{~cm}(v \geq 10 \mathrm{GHz})$, as shown by the dashed blue curve in Figure 8 . The best-fit parameters of the dust component are $\mathrm{T}_{\mathrm{d}}=172 \mathrm{~K}, \beta=2.1, \tau_{1.3 \mathrm{~mm}}=0.86$, and $\theta_{\mathrm{d}}=0$ '.20 $700 \mathrm{au}$. Because the primary aim of our SED modelling is to better understand the nature of the central ionised source, we consider the best-fit dust parameters as indicative, noting in particular the degeneracy between dust temperature and $\beta$ due to having measurements only on the Rayleigh-Jeans portion of the dust emission spectrum. Overlaying the flux densities of Moscadelli et al. (2016) in Figure 8 (open points), we find reasonable consistency at 2.3 and $1.4 \mathrm{~cm}$ considering the difference in uv range, but an excess of emission at $4.8-5.5 \mathrm{~cm}$ compared to our best-fit dust and uniform ionised sphere model.

Given the observed spectral index at these wavelengths ( $\alpha=0.57 \pm 0.63$; Moscadelli et al. 2016), we model this excess as an ionised jet in the form of a truncated power law density profile $\left(F_{v} \propto v^{0.6}\right)$. Fitting to all of the datapoints shown in Figure 8 (and listed in Table 4), we compare three models: (i) a three component model including dust, a uniform density ionised sphere (which we interpret as a hypercompact (HC) $\mathrm{H}$ II region, as discussed below), and an ionised jet; (ii) a two component model including only dust and a uniform density ionised sphere; and (iii) a two component model including only dust and an ionised jet. For the three component model, the dust parameters were fixed to those described above, in light of the large number of free parameters. We first attempted to fit for all six ionised gas parameters (three describing the ionised sphere, and three for the jet), but found that the jet component is not well constrained, especially considering the dispersion in the published flux density measurements at $4.3-5.5 \mathrm{~cm}$, which is due in part to the complexity in the deconvolution of the bright UC HII region (G11.94-0.62) located $\sim 1^{\prime}$ to the north-northeast (Wood \& Churchwell 1989). For this reason, we adopted a nominal central density of $n_{e}=10^{6} \mathrm{~cm}^{-3}$ and electron temperature of $T_{e}=10^{4} \mathrm{~K}$ and fit only for the size of the jet. To assess which of the three models - (i), (ii), or (iii), above - provides the best description of the data, we computed the reduced $\chi^{2}$ for the best-fit model of each class. The three-component model best represents the data, with a reduced $\chi^{2}$ of 1.4, compared to a reduced $\chi^{2}$ of 3.0 for the model with only dust and an ionised sphere, and a reduced $\chi^{2}$ of 7.0 for the model with only dust and an ionised jet. The dust and jet model is shown as a dashed red curve in Figure 8, which illustrates that this model produces a poor overall fit to the SED, failing to reproduce the $\sim 1.3 \mathrm{~cm}$ and $\leq 1.1 \mathrm{~mm}$ emission. For the three-component model, the best-fit ionised gas parameters are electron temperature $T_{e}=9500 \mathrm{~K}$, electron density $n_{e}=5.8 \times 10^{6} \mathrm{~cm}^{-3}$ and radius $R_{e}=21$ au $\left(\sim 00^{\prime} 006\right.$ at a distance of $3.37 \mathrm{kpc}$ ) for the $\mathrm{HC} \mathrm{HII}$ region, and a half-power radius of $17 \mathrm{au}\left(\sim 0{ }^{\prime} 005\right.$ at $\left.3.37 \mathrm{kpc}\right)$ for the jet. As shown in Figure 8, the three-component combined model (whose individual components are shown as dotted lines) produces a reasonable fit to the SED. The modelled source sizes for both the ionised and dust components are consistent with observational constraints, e.g. with CM1 being unresolved in all of our VLA observations and in those of Moscadelli et al. (2016) (see also Section 3.1), and with MM1 being unresolved by the SMA (Cyganowski et al. 2014).

Our modelling results support a picture in which the centimetre-wavelength continuum emission associated with MM1 arises from a very small hypercompact (HC) H II region that is gravitationally 'trapped' by an accretion flow (e.g. Keto 2003, 2007), possibly accompanied by a compact ionised jet. These results are consistent with other evidence for ongoing accretion by the central (proto)star, including the fact that it drives an active outflow (Figure 1). Indeed, the momentum outflow rate estimated by Moscadelli et al. (2016) from VLBA observations of $\mathrm{H}_{2} \mathrm{O}$ masers is exceptionally high, $2 \times 10^{-2} \mathrm{M}_{\odot} \mathrm{yr}^{-1} \mathrm{~km} \mathrm{~s}^{-1}$. A high accretion rate could also explain the moderate luminosity of the region $\left(\sim 10^{4} \mathrm{~L}_{\odot}\right)$ compared to the enclosed mass implied for MM1 by our kinematic modelling (Section 4.1): in evolutionary models of massive (proto)stars, high accretion rates result in large radii and low effective temperatures (e.g. Hosokawa \& Omukai 2009; Hosokawa et al. 2010). Finally, the early evolutionary stage implied by a gravitationally trapped H II region and a swollen, non-ZAMS configuration is consistent with the short dynamical timescale of the outflow driven by MM1, $\$ 10,000$ years (Cyganowski et al. 2011a).

The self-consistent scenario outlined above - in which a high rate of accretion governs many observable characteristics - leads us to favour a $\mathrm{HC} \mathrm{H}$ II region interpretation of the centimetre-wavelength $(\lambda=0.9-3 \mathrm{~cm})$ emission from MM1. We note, however, that $\mathrm{HC} \mathrm{H}$ II regions and ionised winds or jets can be difficult to differentiate observationally. The distinction is in part dynamical - jets have higher velocities than winds (as reflected in larger radio recombination line widths and/or proper motions), which have higher velocities than HC H II regions (e.g. Hoare et al. 2007; Hoare \& Franco 2007). The other distinction is the source of ionising photons: for $\mathrm{HC} \mathrm{H}$ II regions (including ionised accretion flows or discs), photoionisation by the central massive (proto)star dominates, while in jets and winds, shocks may contribute significantly to the ionisation (e.g. Shepherd et al. 2004; Keto 2007; Galván-Madrid et al. 2010). Neither distinguishing property can be directly accessed with existing observations, and both $\mathrm{HC} \mathrm{H}$ II regions and ionised winds or jets are characterised by intermediate centimetre-wavelength spectral indices $\left(\mathrm{S}_{v} \propto v^{\alpha},-0.1<\alpha<2\right)$. Moscadelli et al. (2016) interpret the centimetre-wavelength emission from G11.92-0.61 MM1 as arising from an ionised wind, based largely on their measured centimetre-wavelength spectral indices. The difference in interpretation likely stems from our more extensive wavelength coverage (e.g. into the submillimetre) and the inclusion of a dust component in our modelling: a three-component combined model, including 
dust, an $\mathrm{HC} \mathrm{H}$ II region, and an ionised jet, provides the best description of the observed SED, as discussed above. Moscadelli et al. (2016) also report a slight elongation in their highest-resolution $(1.4 \mathrm{~cm})$ image, but the continuum emission is not well-resolved (see also Section 3.1). Additional, higher angular resolution VLA observations (e.g. at $0.9 \mathrm{~cm}$ and $0.7 \mathrm{~cm}$, in the most-extended A-configuration) are necessary to establish whether or not the ionised component is spatially extended on $\sim 00^{\prime} 1$ scales. Importantly, a contribution to the centimetre-wavelength emission from an ionised wind or jet - as in our three component model (Figure 8) - is entirely consistent with our conclusion that the central source of MM1 is a very young massive (proto)star, characterised by ongoing accretion and a swollen, non-ZAMS configuration.

\subsection{Physical properties of the disc estimated from dust emission}

Our modelling of MM1's centimetre-submillimetre wavelength SED confirms that the observed $1.3 \mathrm{~mm}$ flux density is dominated by thermal dust emission: in our best-fit model, dust accounts for 99.5 per cent of the emission at $1.3 \mathrm{~mm}$. We estimate a gas mass for the disc from the measured $1.3 \mathrm{~mm}$ integrated flux density using a simple model of isothermal dust emission, corrected for dust opacity (Cyganowski et al. 2011a, Equation 3). As in Cyganowski et al. (2014), these estimates assume a gas-to-dust mass ratio of 100 and a dust opacity $\kappa_{1.3 \mathrm{~mm}}=1.1 \mathrm{~cm}^{2} \mathrm{~g}^{-1}$ (for grains with thin ice mantles and coagulation at $10^{8} \mathrm{~cm}^{-3}$; Ossenkopf \& Henning 1994). For these estimates, we adopt the average temperatures of the cool and warm components from our pixelby-pixel $\mathrm{CH}_{3} \mathrm{CN}$ modelling, $153 \pm 10 \mathrm{~K}$ and $227 \pm 14 \mathrm{~K}$ (Section 4.2 ; the quoted uncertainty is the standard deviation over all fitted pixels), and the best-fit dust temperature from Section 4.3 of $172 \mathrm{~K}$. As these temperatures are very similar to the range adopted by Cyganowski et al. (2014) based on fitting the $\mathrm{CH}_{3} \mathrm{CN}$ spectrum at the millimetre continuum peak, the mass estimates are nearly identical to those in Cyganowski et al. (2014): $\mathrm{M}_{\text {gas }}=3.3 \mathrm{M}_{\odot}, 2.9 \mathrm{M}_{\odot}$, and $2.1 \mathrm{M}_{\odot}$ for $\mathrm{T}_{\text {dust }}=153 \mathrm{~K}, 172 \mathrm{~K}$, and $227 \mathrm{~K}$, respectively. We note that even if the centimetre-wavelength continuum emission arose entirely from an ionised wind (e.g. Moscadelli et al. 2016) with $\alpha=0.6$ out to millimetre wavelengths, the contribution to the $1.3 \mathrm{~mm}$ flux density would be negligible for our mass estimates ( $\sim 1 \mathrm{mJy}$ contribution at $1.3 \mathrm{~mm}$, corresponding to a difference in the estimated mass of $\sim 0.02-0.04 M_{\odot}$, depending on $\left.\mathrm{T}_{\text {dust }}\right)$.

Calculating corresponding $\mathrm{H}_{2}$ number densities for a cylindrical, rather than a spherical, geometry yields estimates of $\mathrm{n}_{\mathrm{H}_{2}} \sim 2-3 \times 10^{10} \mathrm{~cm}^{-3}$ for a characteristic disc height of $7 \mathrm{au}$ and $\mathrm{n}_{\mathrm{H}_{2}} \sim 5-8 \times 10^{9} \mathrm{~cm}^{-3}$ for a disc height of $30 \mathrm{au}$ (measured from the midplane). We emphasise that these estimated number densities are averages over the entire disc, based on an isothermal estimate of the gas mass associated with the observed dust emission - which, as discussed in Section 3.3, is likely more compact than the gas disc. We also note that while the calculated dust opacities, estimated as $\tau_{\text {dust }}=-\ln \left(1-T_{\mathrm{b}} / T_{\text {dust }}\right)$, are moderate $(0.2-0.3$, comparable to the estimates in Cyganowski et al. 2014), these are similarly averages over the entire source, and do not capture variations in opacity within the disc (e.g. associated with a dense midplane).

\subsection{G11.92-0.61 MM1 in context}

The results of our kinematic fitting (Section 4.1) suggest that G11.92-0.61 MM1 may be the most massive proto-O star to date with strong evidence for the presence of a Keplerian disc. Of candidates reported in the literature, only AFGL 2591-VLA3 has a comparable central source mass: $\sim 40 \mathrm{M}_{\odot}$ (L $2 \times 10^{5} \mathrm{~L}_{\odot}$; Jiménez-Serra et al. 2012; Sanna et al. 2012), compared to $\sim 30-60 \mathrm{M}_{\odot}$ for MM1. Studies of other proto$\mathrm{O}$ stars with evidence for Keplerian discs find enclosed or central source masses of $<30 \mathrm{M}_{\odot}$. For NGC6334I(N)-SMA $1 \mathrm{~b}$, perhaps the closest analogue to G11.92-0.61 based on IR properties, Hunter et al. (2014) find an enclosed mass of $10-30 \mathrm{M}_{\odot}$. Interestingly, IRAS 16547-4247 and AFGL 4176 - regions with luminosities 6-10× that of G11.92-0.61 $\left(\sim 10^{4} \mathrm{~L}_{\odot}\right.$; Cyganowski et al. 2011a; Moscadelli et al. 2016) also have enclosed or central source masses of $<30 \mathrm{M}_{\odot}$ (Zapata et al. 2015; Johnston et al. 2015). Taken at face value, this collection of results suggests that enclosed mass does not scale directly with luminosity for proto-O stars. However, the differences between the estimated luminosities of different sources are comparable to the uncertainties in the estimates, particularly for sources that do not have maser parallax distances (e.g. IRAS 16547-4247 and AFGL 4176).

In contrast, our estimated (gas) mass for the MM1 disc is similar to the mass estimates obtained for other candidate discs around proto-O stars using similar dust properties. In the context of comparing the small number of discs around O-type (proto)stars, it is worth noting the substantial uncertainty in mass estimates associated with the (assumed) dust opacity, $\kappa_{v}$. Our estimates above (like those of Wang et al. 2012; Hunter et al. 2014; Zapata et al. 2015) adopt dust opacities from Ossenkopf \& Henning (1994) for grains with ice mantles. For Draine (2003) interstellar grains, $\kappa_{1.2 \mathrm{~mm}}$ is lower by a factor of $\gtrsim 5$ (for $\mathrm{R}_{V}=5.5$, as adopted by Johnston et al. 2015), yielding mass estimates that are larger by the same factor. This difference in assumed dust properties accounts for the much larger mass reported by Johnston et al. (2015) for the AFGL $4176 \operatorname{disc}\left(\sim 12 \mathrm{M}_{\odot}\right)$, compared to the values of a few solar masses $\left(\sim 2-6 \mathrm{M}_{\odot}\right)$ characteristic of MM1 and other massive disc candidates (e.g. Wang et al. 2012; Hunter et al. 2014; Zapata et al. 2015).

The relatively large disc-to-star mass ratio derived from our observations of G11.92-0.61 MM1 ( $\gtrsim 0.035)$ indicates that self-gravity may play a role in the evolution of the disc. Self-gravitating discs are efficient transporters of angular momentum, and hence provide a suitable means of assembling relatively massive stars on short timescales. In a companion paper (Forgan et al, submitted) we compute simple semi-analytic models of self-gravitating discs (Forgan \& Rice 2011, 2013), both for MM1 and for other massive Keplerian disc candidates recently observed. We find that these simple models provide reasonably good estimates of the observed disc mass, given the observational constraints on the disc inner and outer radii, stellar mass and accretion rate. Most intriguingly, the models predict that the disc around MM1 should be sufficiently unstable to fragment into low mass protostars. These objects are beyond the resolution of 


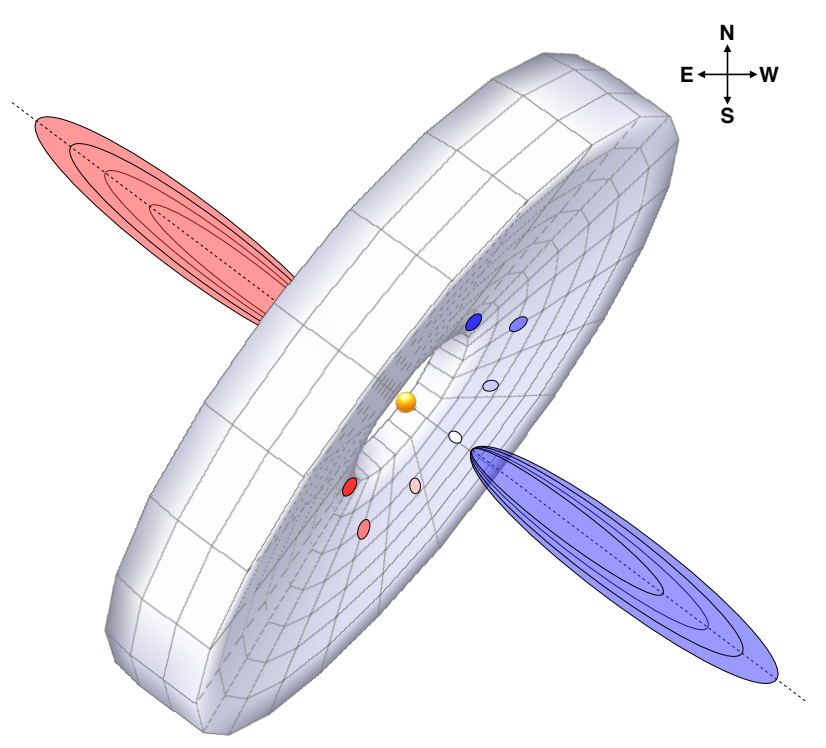

Figure 9. A proposed morphology for the immediate vicinity of MM1 as viewed from Earth. The maximum velocity gradient obtained from the line channel analysis (red-blue points) lies perpendicular to the axis of the molecular outflow (Fig. 1). The closed loop structure of the channel centroids (Fig. 6) for many of the emission lines can be explained by a flared, optically thick Keplerian disc, viewed at an intermediate inclination. Such a disc would also exhibit a hot spot in temperature towards the South-West (Fig. 7).

our observations at this time, but may be detectable with e.g. ALMA.

\section{CONCLUSIONS}

In this paper, we have used sub-arcsecond ( $\$ 1550 \mathrm{au})$ resolution SMA $1.3 \mathrm{~mm}$ and VLA 3.0 and $0.9 \mathrm{~cm}$ observations of the high-mass (proto)star G11.92-0.61 MM1 to characterise the nature of the central source and examine the kinematics and physical conditions of the gas surrounding it. Our main findings are:

- The compact molecular line emission associated with the MM1 millimetre continuum source exhibits a velocity gradient that is approximately perpendicular to the highvelocity bipolar molecular outflow driven by MM1. The velocity gradient of the compact gas is remarkably consistent, being seen in lines of varying excitation energy and in 10 different chemical species, including $\mathrm{CH}_{3} \mathrm{OH}, \mathrm{CH}_{3} \mathrm{OCHO}$, OCS, $\mathrm{HNCO}, \mathrm{CH}_{3} \mathrm{CH}_{2} \mathrm{CN}, \mathrm{H}_{2} \mathrm{CO}$ and DCN.

- From the $8 \mathrm{GHz}$ of bandwidth covered by our SMA observations, we identify 15 potentially 'disc tracing' lines. For all of these lines, position-velocity diagrams exhibit the characteristic pattern expected for a Keplerian disc: the highest velocities are seen nearest the central source, and the most spatially extended emission at lower velocities.

- Our kinematic modelling of the MM1 disc yields a bestfit enclosed mass of $60_{-27}^{+21} \mathrm{M}_{\odot}$ and disc inclination of $35_{-6}^{+20}$ 。 (to the line of sight), using all 'disc-tracing' lines other than $\mathrm{CH}_{3} \mathrm{OH}$ (nine transitions). The six 'disc-tracing' transitions of $\mathrm{CH}_{3} \mathrm{OH}$ exhibit a somewhat different morphology than the rest of the lines in plots of emission centroid position as a function of velocity, and were fit separately. The best fitting disc model for the $\mathrm{CH}_{3} \mathrm{OH}$ transitions yields a disc enclosing a mass of $34_{-12}^{+28} \mathrm{M}_{\odot}$ at an inclination of $52_{-14}^{+11}$. Together these results imply a central source mass of $\sim 30-60 \mathrm{M}_{\odot}$, making MM1 potentially the most massive proto-O star with strong evidence for a Keplerian disc.

- For many observed molecular lines, the extent of the gas emission is larger than that of the dust continuum emission from the disc. From our kinematic modelling of the gas emission, we find a disc outer radius of $1200 \mathrm{au}$.

- Two temperature components are required to fit the $\mathrm{CH}_{3} \mathrm{CN} \mathrm{J}=12-11$ emission from the MM1 disc using an LTE radiative transfer model. The cooler $(153 \pm 10 \mathrm{~K})$ and warmer $(227 \pm 14 \mathrm{~K})$ components may correspond to $\mathrm{CH}_{3} \mathrm{CN}$ reservoirs in different disc layers, as in recent models of discs around low-mass protostars (Walsh et al. 2015).

- Our modelling of the centimetre-submillimetre wavelength SED of MM1 confirms that the observed $1.3 \mathrm{~mm}$ flux density is dominated by dust. Applying a simple model of isothermal dust emission, we estimate a disc gas mass of $2.1-3.3 \mathrm{M}_{\odot}\left(\right.$ for $\left.\mathrm{T}_{\text {dust }}=227-153 \mathrm{~K}\right)$. This mass estimate is similar to those for most other candidate Keplerian discs around proto-O stars that assume similar dust properties. MM1's relatively high disc-to-star mass ratio ( $\gtrsim 0.035)$ suggests that the disc may be self-gravitating, which we explore in a companion modelling paper (Forgan et al., submitted).

- From our SED modelling, we find that the centimetrewavelength flux density of MM1 is dominated by free-free emission. We model the free-free and dust emission simultaneously, and find that the ionised gas is best-fit by a model of a uniform density ionised sphere with electron temperature $9500 \mathrm{~K}$, electron density $5.8 \times 10^{6} \mathrm{~cm}^{-3}$ and radius $21 \mathrm{au}$. These properties are consistent with a very small, gravitationally trapped HC HiI region, possibly accompanied by a compact ionised jet.

In combination, our results suggest that G11.92-0.61 MM1 is likely a young proto-O star, in a swollen, non-ZAMS configuration, surrounded by a Keplerian disc with a morphology similar to that shown in Figure 9. Our observations and modelling support a self-consistent picture in which accretion is ongoing, and a high accretion rate governs many observable properties, including the presence of a gravitationally trapped HC HII region and a moderate luminosity $\left(\sim 10^{4} \mathrm{~L}_{\odot}\right)$ for a massive $\left(\sim 30-60 \mathrm{M}_{\odot}\right)$ central star. Future higher angular resolution observations will be required to spatially resolve the protostar-disc system. Such observations will be essential to compare the role and physics of disc accretion in high- and low-mass star formation, and to develop a clearer understanding of the accretion processes at work in massive young stellar objects.

\section{ACKNOWLEDGEMENTS}

We would like to thank Melvin Hoare, Simon Purser and Katharine Johnston for helpful discussions regarding the nature of the centimetre-wavelength emission, and Catherine 
Walsh for kindly providing further information on the behaviour of $\mathrm{CH}_{3} \mathrm{CN}$ in her chemical models. We also thank Kenny Wood, Claire Davies, and Christine Koepferl for their input at an early stage of this project, and the referee for a constructive report.

JDI gratefully acknowledges support from the DISCSIM project, grant agreement 341137, funded by the European Research Council under ERC-2013-ADG. CJC acknowledges support from STFC grant ST/M001296/1. PN, CJC and JDI gratefully acknowledge support in the form of an Undergraduate Research Bursary from the Royal Astronomical Society. DF acknowledges support from the ECOGAL project, grant agreement 291227, funded by the European Research Council under ERC-2011-ADG. This research has made use of NASA's Astrophysics Data System Bibliographic Services; Astropy, a community-developed core Python package for Astronomy (Astropy Collaboration et al. 2013); APLpy, an open-source plotting package for Python hosted at http://aplpy.github.com, and the CASSIS software and VADMC databases (http://www.vamdc.eu/). CASSIS has been developed by IRAP-UPS/CNRS (http://cassis. irap.omp.eu). The Submillimeter Array is a joint project between the Smithsonian Astrophysical Observatory and the Academia Sinica Institute of Astronomy and Astrophysics, and is funded by the Smithsonian Institution and the Academia Sinica. The National Radio Astronomy Observatory is a facility of the National Science Foundation operated under agreement by the Associated Universities, Inc.

\section{References}

Alexander R. D., Clarke C. J., Pringle J. E., 2006, MNRAS, 369, 229

Araya E., Hofner P., Kurtz S., Bronfman L., DeDeo S., 2005, ApJS, 157, 279

Astropy Collaboration et al., 2013, A\&A, 558, A33

Beltrán M. T., de Wit W. J., 2016, A\&ARv, 24, 6

Beltrán M. T., Cesaroni R., Neri R., Codella C., 2011, A\&A, 525, A151

Beltrán M. T., et al., 2014, A\&A, 571, A52

Benedettini M., et al., 2013, MNRAS, 436, 179

Beuther H., Walsh A. J., 2008, ApJ, 673, L55

Bik A., Thi W. F., 2004, A\&A, 427, L13

Boley P. A., et al., 2013, A\&A, 558, A24

Breen S. L., Ellingsen S. P., 2011, MNRAS, 416, 178

Brogan C. L., Chandler C. J., Hunter T. R., Shirley Y. L., Sarma A. P., 2007, ApJ, 660, L133

Ceccarelli C., Hollenbach D. J., Tielens A. G. G. M., 1996, ApJ, 471,400

Cesaroni R., 2005, Ap\&SS, 295, 5

Cesaroni R., Galli D., Lodato G., Walmsley M., Zhang Q., 2006, Nature, 444, 703

Cesaroni R., Galli D., Lodato G., Walmsley C. M., Zhang Q., 2007, Protostars and Planets V, pp 197-212

Cesaroni R., Beltrán M. T., Zhang Q., Beuther H., Fallscheer C., 2011, A\&A, 533, A73

Cesaroni R., Galli D., Neri R., Walmsley C. M., 2014, A\&A, 566, A73

Comito C., Schilke P., Endesfelder U., Jiménez-Serra I., MartínPintado J., 2007, A\&A, 469, 207

Cyganowski C. J., et al., 2008, AJ, 136, 2391

Cyganowski C. J., Brogan C. L., Hunter T. R., Churchwell E., 2009, ApJ, 702, 1615
Cyganowski C. J., Brogan C. L., Hunter T. R., Churchwell E., Zhang Q., 2011a, ApJ, 729, 124

Cyganowski C. J., Brogan C. L., Hunter T. R., Churchwell E., 2011b, ApJ, 743, 56

Cyganowski C. J., et al., 2014, ApJ, 796, L2

Davies B., Lumsden S. L., Hoare M. G., Oudmaijer R. D., de Wit W.-J., 2010, MNRAS, 402, 1504

Davies B., Hoare M. G., Lumsden S. L., Hosokawa T., Oudmaijer R. D., Urquhart J. S., Mottram J. C., Stead J., 2011, MNRAS, 416, 972

Draine B. T., 2003, ARA\&A, 41, 241

Forgan D., Rice K., 2011, MNRAS, 417, 1928

Forgan D., Rice K., 2013, MNRAS, 430, 2082

Galván-Madrid R., Zhang Q., Keto E., Ho P. T. P., Zapata L. A., Rodríguez L. F., Pineda J. E., Vázquez-Semadeni E., 2010, ApJ, 725, 17

Gordon M. A., 1995, A\&A, 301, 853

Guilloteau S., Dutrey A., Piétu V., Boehler Y., 2011, A\&A, 529, A105

Harries T. J., Haworth T. J., Acreman D. M., 2014, Astrophysics and Space Science Proceedings, 36, 395

Hernández-Hernández V., Zapata L., Kurtz S., Garay G., 2014, ApJ, 786, 38

Hoare M. G., Franco J., 2007, Astrophysics and Space Science Proceedings, 1,61

Hoare M. G., Kurtz S. E., Lizano S., Keto E., Hofner P., 2007, Protostars and Planets V, pp 181-196

Hofner P., Churchwell E., 1996, A\&AS, 120, 283

Hosokawa T., Omukai K., 2009, ApJ, 691, 823

Hosokawa T., Yorke H. W., Omukai K., 2010, ApJ, 721, 478

Hughes A. M., Wilner D. J., Andrews S. M., Qi C., Hogerheijde M. R., 2011, ApJ, 727, 85

Hunter T. R., Brogan C. L., Cyganowski C. J., Young K. H., 2014, ApJ, 788, 187

Ilee J. D., et al., 2013, MNRAS, 429, 2960

Ilee J. D., Fairlamb J., Oudmaijer R. D., Mendigutía I., van den Ancker M. E., Kraus S., Wheelwright H. E., 2014, MNRAS, 445,3723

Jiménez-Serra I., Zhang Q., Viti S., Martín-Pintado J., de Wit W.-J., 2012, ApJ, 753, 34

Johnston K. G., Beuther H., Linz H., Boley P., Robitaille T. P., Keto E., Wood K., van Boekel R., 2014, Astrophysics and Space Science Proceedings, 36, 413

Johnston K. G., et al., 2015, ApJ, 813, L19

Kaufman M. J., Hollenbach D. J., Tielens A. G. G. M., 1998, ApJ, 497, 276

Keto E., 2003, ApJ, 599, 1196

Keto E., 2007, ApJ, 666, 976

Klassen M., Pudritz R., Kuiper R., Peters T., Banerjee R., 2016, preprint, (arXiv:1603.07345)

Kraus S., et al., 2010, Nature, 466, 339

Krumholz M. R., Klein R. I., McKee C. F., Offner S. S. R., Cunningham A. J., 2009, Science, 323, 754

Kuiper R., Klahr H., Beuther H., Henning T., 2010, ApJ, 722, 1556

Kuiper R., Klahr H., Beuther H., Henning T., 2011, ApJ, 732, 20

Lee H.-T., Takami M., Duan H.-Y., Karr J., Su Y.-N., Liu S.-Y., Froebrich D., Yeh C. C., 2012, ApJS, 200, 2

Lee H.-T., et al., 2013, ApJS, 208, 23

Maret S., 2015, thindisk: Thindisk v1.0, doi:10.5281/zenodo.13823, http://dx.doi.org/10.5281/ zenodo. 13823

Moscadelli L., et al., 2016, A\&A, 585, A71

Mottram J. C., et al., 2011, ApJ, 730, L33

Olnon F. M., 1975, A\&A, 39, 217

Ossenkopf V., Henning T., 1994, A\&A, 291, 943

Pankonin V., Churchwell E., Watson C., Bieging J. H., 2001, ApJ, 558,194 
Patel N. A., et al., 2005, Nature, 437, 109

Pérez L. M., et al., 2012, ApJ, 760, L17

Qiu K., Zhang Q., Beuther H., Fallscheer C., 2012, ApJ, 756, 170

Rathborne J. M., Jackson J. M., Chambers E. T., Stojimirovic I.,

Simon R., Shipman R., Frieswijk W., 2010, ApJ, 715, 310

Sánchez-Monge Á., et al., 2013, A\&A, 552, L10

Sanna A., Reid M. J., Carrasco-González C., Menten K. M., Brunthaler A., Moscadelli L., Rygl K. L. J., 2012, ApJ, 745, 191

Sato M., et al., 2014, ApJ, 793, 72

Shepherd D. S., Kurtz S. E., Testi L., 2004, ApJ, 601, 952

Shimajiri Y., et al., 2015, ApJS, 221, 31

Walsh C., et al., 2014, ApJ, 791, L6

Walsh C., Nomura H., van Dishoeck E., 2015, A\&A, 582, A88

Wang K.-S., van der Tak F. F. S., Hogerheijde M. R., 2012, A\&A, 543, A22

Wheelwright H. E., Oudmaijer R. D., de Wit W. J., Hoare M. G., Lumsden S. L., Urquhart J. S., 2010, MNRAS, 408, 1840

Wood D. O. S., Churchwell E., 1989, ApJS, 69, 831

Yorke H. W., Sonnhalter C., 2002, ApJ, 569, 846

Zapata L. A., Palau A., Galván-Madrid R., Rodríguez L. F., Garay G., Moran J. M., Franco-Hernández R., 2015, MNRAS, 447, 1826

de Gregorio-Monsalvo I., et al., 2013, A\&A, 557, A133

This paper has been typeset from a $\mathrm{T}_{\mathrm{E}} \mathrm{X} / \mathrm{LAT} \mathrm{T} \mathrm{X}$ file prepared by the author. 